CONFORMAL GEOMETRY AND DYNAMICS

An Electronic Journal of the American Mathematical Society

Volume 8, Pages 1-35 (February 24, 2004)

S 1088-4173(04)00103-1

\title{
PARAMETRIZED DYNAMICS OF THE WEIERSTRASS ELLIPTIC FUNCTION
}

\author{
JANE HAWKINS AND LORELEI KOSS
}

\begin{abstract}
We study parametrized dynamics of the Weierstrass elliptic $\wp$ function by looking at the underlying lattices; that is, we study parametrized families $\wp_{\Lambda}$ and let $\Lambda$ vary. Each lattice shape is represented by a point $\tau$ in a fundamental period in modular space; for a fixed lattice shape $\Lambda=[1, \tau]$ we study the parametrized space $k \Lambda$. We show that within each shape space there is a wide variety of dynamical behavior, and we conduct a deeper study into certain lattice shapes such as triangular and square. We also use the invariant pair $\left(g_{2}, g_{3}\right)$ to parametrize some lattices.
\end{abstract}

\section{INTRODUCTION}

It is well known that transcendental meromorphic functions such as the exponential, sine, and tangent families exhibit dynamical behavior both similar to and distinct from that of rational maps, as studied in [1], 2], 3], 4], [5], 13], 14], [15], and $[18$ and the references therein. In an earlier paper the authors give examples and results about the dynamics of the Weierstrass elliptic $\wp$ function considered as a meromorphic function of $\mathbb{C}[9]$. What sets this family of transcendental maps apart from many of the others studied is the rich algebraic structure of elliptic functions which influences the dynamics in diverse ways. For example, we can give precise formulas for the generators of lattices $\Lambda$ giving rise to Weierstrass elliptic functions with certain prescribed Fatou components; there are many theorems of this nature in 9] and in this paper.

Let $\wp_{\Lambda}$ denote the Weierstrass elliptic $\wp$ function based on the lattice $\Lambda$; it is shown in [9] that the measure theoretic and dynamical properties of $\wp_{\Lambda}$ depend on $\Lambda$. Moreover, even for a fixed shape lattice such as square, the dynamics vary widely. We give examples of rectangular square lattices (generated by vectors $\lambda$ and $\lambda i$ for some $\lambda>0$ ), some with superattracting fixed points and others with Julia set the whole sphere. In 9 we focused on lattices $\Lambda$ such that $\bar{\Lambda}=\Lambda$, i.e., real lattices; since one of the critical points and many of the critical values are real in this case, this restriction provided useful tools for the study. However, many general results about the dynamics of elliptic functions can be proved without restricting to real lattices or to the Weierstrass $\wp$ function (see for example [14]).

Received by the editors May 21, 2003 and, in revised form, January 23, 2004.

2000 Mathematics Subject Classification. Primary 37F45.

Key words and phrases. Complex dynamics, meromorphic functions, Julia sets, holomorphic families, parameter space.

The second author was supported in part by NSF Grant 9970575. 
We continue the study here by looking at parameter space for Weierstrass elliptic $\wp$ functions. Since lattices in $\mathbb{C}$ are parametrized by a pair of linearly independent vectors in $\mathbb{R}^{2}$, and since there are infinitely many ways to generate the same lattice, we can make some reductions in parameter space. It is enough to restrict attention to one fundamental period for the modular group, and then each point in that region gives rise to a parameter space corresponding to one shape of lattice.

While some of our results are concentrated on specific shapes, namely square and triangular lattices, which represent only two points in modular space, many of the results extend to arbitrary lattices and other elliptic functions.

The paper is organized as follows. In Section 2 we give some background on the dynamics of meromorphic functions and elliptic functions and then in Section 3 we discuss some properties of lattices which form the periods of elliptic functions. In Section 4 we discuss the links between the lattice, the corresponding Weierstrass $\wp$ function, and its postcritical set. In particular, the algebraic invariants $g_{2}$ and $g_{3}$ are closely related to the postcritical set through standard elliptic identities and hence carry information about the dynamics of $\wp$. In Section 5 we turn to the connection of these properties to the Fatou and Julia sets of $\wp$, denoted $F(\wp)$ and $J(\wp)$. It was shown by Kotus and Urbanski 14 that for any lattice $\Lambda$, since $\wp_{\Lambda}$ is an elliptic function of order $2, J\left(\wp_{\Lambda}\right)$ has Hausdorff dimension greater than $4 / 3$.

In Section 5 we also introduce a new topological type of Fatou component arising from the periodicity of $\wp$ and hence of $J(\wp)$ and $F(\wp)$. Starting with $\wp$ based on the lattice $\Lambda \subset \mathbb{C}$, a component $F_{o} \subset F(\wp)$ is called a toral band if the projection of $F_{o}$ to the torus $\mathbb{C} / \Lambda$ is (or contains) a band around the torus. Additionally in Section 5 we prove that the Fatou set of any Weierstrass elliptic $\wp$ function contains no wandering domains, Baker domains, or Herman rings.

In Section [6 we discuss parameterizing the Weierstrass elliptic functions according to the underlying lattice. We prove results allowing us to view a reduced holomorphic family of maps in this space. In Sections 7 [9 we prove results about parameter space in general, and for specific shapes. We give some detailed results about parameter space for triangular, square, and real lattices. We prove the existence of lattices which give rise to maps with superattracting fixed points or cycles as well as those with Julia set the whole sphere. In Section 10 we give a sufficient condition for the existence of an attracting toral band and show the existence of an attracting toral band for a rectangular lattice. In Section 11 we address some remaining open problems. So far we cannot show that any Cantor Julia set exists for a Weierstrass $\wp$ function, though for other elliptic functions we have some examples. Similarly, we show how to obtain Siegel disks for elliptic functions that are not restricted to the Weierstrass $\wp$ function.

The authors would like to thank the referee for helpful comments and criticisms of an earlier version of this paper and for pointing out the Siegel disk construction in Section [11,

\section{ITERATION OF MEROMORPHIC FUNCTIONS}

Let $f: \mathbb{C} \rightarrow \mathbb{C}_{\infty}$ be a meromorphic function where $\mathbb{C}_{\infty}=\mathbb{C} \cup\{\infty\}$ is the Riemann sphere. The Fatou set $F(f)$ is the set of points $z \in \mathbb{C}_{\infty}$ such that $\left\{f^{n}: n \in \mathbb{N}\right\}$ is defined and normal in some neighborhood of $z$. The Julia set is the complement of the Fatou set on the sphere, $J(f)=\mathbb{C}_{\infty} \backslash F(f)$. Notice that $\mathbb{C}_{\infty} \backslash \overline{\bigcup_{n \geq 0} f^{-n}(\infty)}$ is the largest open set where all iterates are defined. If $f$ has at least one pole that 
is not an omitted value, then $\overline{\bigcup_{n>0} f^{-n}(\infty)}$ has more than two elements. Since $f\left(\mathbb{C}_{\infty} \backslash \overline{\bigcup_{n \geq 0} f^{-n}(\infty)}\right) \subset \mathbb{C}_{\infty} \backslash \overline{\bigcup_{n \geq 0} f^{-n}(\infty)}$, Montel's theorem implies that

$$
J(f)=\overline{\bigcup_{n \geq 0} f^{-n}(\infty)} .
$$

Let $C r i t(f)$ denote the set of critical points of $f$, i.e.,

$$
\operatorname{Crit}(f)=\left\{z: f^{\prime}(z)=0\right\} \text {. }
$$

If $z_{0}$ is a critical point, then $f\left(z_{0}\right)$ is a critical value. We say that $w$ is an asymptotic value of $f$ if there exists a path $\alpha:[0, \infty) \rightarrow \mathbb{C}$ such that $\lim _{t \rightarrow \infty}|\alpha(t)|=\infty$ and $\lim _{t \rightarrow \infty} f(\alpha(t))=w$. The singular set $\operatorname{Sing}(f)$ of $f$ is the set of critical and finite asymptotic values of $f$ and their limit points. A function is called Class $S$ if $f$ has only finitely many critical and asymptotic values. The postcritical set of $f$ is:

$$
P(f)=\overline{\bigcup_{n \geq 0} f^{n}(\operatorname{Sing}(f))} \text {. }
$$

A point $z_{0}$ is periodic of period $p$ if there exists a $p \geq 1$ such that $f^{p}\left(z_{0}\right)=z_{0}$. We also call the set $\left\{z_{0}, f\left(z_{0}\right), \ldots, f^{p-1}\left(z_{0}\right)\right\}$ a $p$-cycle. The multiplier of a point $z_{0}$ of period $p$ is the derivative $\left(f^{p}\right)^{\prime}\left(z_{0}\right)$. A periodic point $z_{0}$ is called attracting, repelling, or neutral if $\left|\left(f^{p}\right)^{\prime}\left(z_{0}\right)\right|$ is less than, greater than, or equal to 1 respectively. If $\left|\left(f^{p}\right)^{\prime}\left(z_{0}\right)\right|=0$, then $z_{0}$ is called a superattracting periodic point. As in the case of rational maps, the Julia set is the closure of the repelling periodic points [1].

Suppose $U$ is a connected component of the Fatou set. We say that $U$ is preperiodic if there exists $n>m \geq 0$ such that $f^{n}(U)=f^{m}(U)$, and the minimum of $n-m=p$ for all such $n, m$ is the period of the cycle. A component of $F$ which is not preperiodic is called a wandering domain.

The classification of periodic components of the Fatou set of a meromorphic function is slightly more complicated than that of rational maps of the sphere, but not in the setting of this paper. Periodic components of $F(f)$ may be attracting domains, parabolic domains, Siegel disks, Herman rings, or Baker domains [1]. The definitions of the first four types of behavior are the same as for rational maps of the sphere. A periodic component $U$ of period $p$ is called a Baker domain if there exists a $z_{0} \in \partial U$ such that $f^{n p}(z) \rightarrow z_{0}$ for $z \in U$ as $n \rightarrow \infty$, but $f^{p}\left(z_{0}\right)$ is not defined.

If $f$ is Class $S$, then $f$ does not have wandering domains [3] or Baker domains [8]. Thus the classification of periodic components of the Fatou set of meromorphic functions which are Class $S$ is no more complicated than that of rational maps of the sphere.

Let $C=\left\{U_{0}, U_{1}, \ldots U_{p-1}\right\}$ be a periodic cycle of components of $F(f)$. If $C$ is a cycle of immediate attractive basins or parabolic domains, then $U_{j} \cap \operatorname{Sing}(f) \neq \emptyset$ for some $0 \leq j \leq p-1$. If $C$ is a cycle of Siegel disks or Herman rings, then $\partial U_{j} \subset \overline{\bigcup_{n \geq 0} f^{n}(\operatorname{Sing}(f))}$ for all $0 \leq j \leq p-1$. In particular, singular points are required for any type of preperiodic Fatou component. 


\section{LATtiCES IN THE PLANE}

3.1. Lattices and fundamental regions. Let $\lambda_{1}, \lambda_{2} \in \mathbb{C} \backslash\{0\}$ such that $\lambda_{2} / \lambda_{1} \notin$ $\mathbb{R}$. We define a lattice of points in the complex plane by

$$
\Lambda=\left[\lambda_{1}, \lambda_{2}\right]:=\left\{m \lambda_{1}+n \lambda_{2}: m, n \in \mathbb{Z}\right\} .
$$

It is well known that two different sets of vectors can generate the same lattice $\Lambda$. If $\Lambda=\left[\lambda_{1}, \lambda_{2}\right]$, then any other generators $\lambda_{3}, \lambda_{4}$ of $\Lambda$ are obtained by multiplying the vector $\left(\lambda_{1}, \lambda_{2}\right)$ by the matrix

$$
A=\left(\begin{array}{ll}
a & b \\
c & d
\end{array}\right)
$$

with $a, b, c, d \in \mathbb{Z}$ and $a d-b c=1$.

We can view $\Lambda$ as a group acting on $\mathbb{C}$ by translation, each $\omega \in \Lambda$ inducing the transformation of $\mathbb{C}$ :

$$
T_{\omega}: z \mapsto z+\omega
$$

Definition 3.1. A closed, connected subset $Q$ of $\mathbb{C}$ is defined to be a fundamental region for $\Lambda$ if:

(1) for each $z \in \mathbb{C}, Q$ contains at least one point in the same $\Lambda$-orbit as $z$;

(2) no two points in the interior of $Q$ are in the same $\Lambda$-orbit.

If $Q$ is any fundamental region for $\Lambda$, then for any $s \in \mathbb{C}$, the set

$$
Q+s=\{z+s: z \in Q\}
$$

is also a fundamental region. Usually (but not always) we choose $Q$ to be a parallelogram, in which case we call $Q$ a period parallelogram for $\Lambda$.

3.2. Shapes of lattices and the modular group. Frequently we refer to types of lattices by the shapes of the corresponding period parallelograms; the word shape has a precise meaning for lattices which we give here. For a lattice $\Lambda=\left[\lambda_{1}, \lambda_{2}\right]$, its appearance is determined by the ratio $\tau=\lambda_{2} / \lambda_{1}$. (We generally choose the generators so that $\operatorname{Im}(\tau)>0$.) If $\Lambda=\left[\lambda_{1}, \lambda_{2}\right]$, and $k \neq 0$ is any complex number, then $k \Lambda$ is also a lattice defined by taking $k \lambda$ for each $\lambda \in \Lambda ; k \Lambda$ is said to be similar to $\Lambda$. For example, the lattice $\Lambda_{\tau}=[1, \tau]$ is similar to the lattice $\Lambda=\lambda_{1} \Lambda_{\tau}$. Similarity is an equivalence relation between lattices, and an equivalence class of lattices is called a shape.

However, for an arbitrary lattice $\Lambda$ presented without generators, $\tau$ is not uniquely determined; changing to new generators for $\Lambda$ via the matrix

$$
A=\left(\begin{array}{ll}
a & b \\
c & d
\end{array}\right)
$$

with $a, b, c, d \in \mathbb{Z}$ and $a d-b c= \pm 1$, induces a map on $\tau$, taking it to $\tau^{\prime}=\frac{a \tau+b}{c \tau+d}$. Adopting the convention that $\operatorname{Im}(\tau)>0$, we only need $a d-b c=1$; the maps of the form $\tau \mapsto \tau^{\prime}$ defined this way form the modular group $\Gamma$.

Indeed the action of the classical modular group $\Gamma$ acting in the upper half plane gives the relation between the possible choices of $\tau$ for one shape (see [7]). A fundamental region for $\Gamma$ is a simply connected set in the upper half plane containing exactly one point from the $\Gamma$-orbit of every $\tau$. 


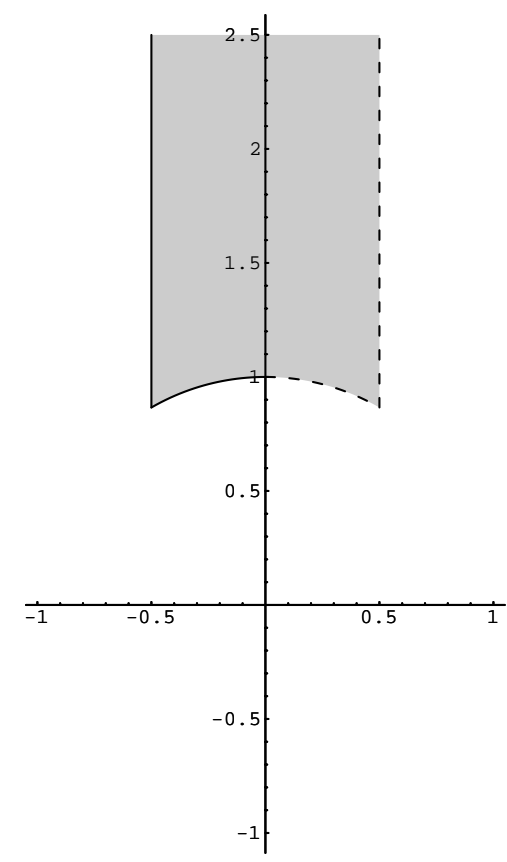

FIgURE 1. The primitive fundamental region

We consider the primitive fundamental region defined by:

$$
\begin{aligned}
B=\{\tau & \in \mathbb{C}: \operatorname{Im}(\tau)>0, \\
& \left.-\frac{1}{2} \leq \operatorname{Re}(\tau)<\frac{1}{2},|\tau| \geq 1, \quad \text { with } \quad|\tau|>1 \quad \text { if } \quad \operatorname{Re}(\tau)>0\right\} .
\end{aligned}
$$

Then every shape is represented by exactly one point in $B$. The region $\mathrm{B}$ is shown in Figure 1

Definition 3.2. (1) We say that $\Lambda=\left[\lambda_{1}, \lambda_{2}\right]$ is real rectangular if there exist generators such that $\lambda_{1}$ is real and $\lambda_{2}$ is purely imaginary. Any lattice similar to a real rectangular lattice is rectangular.

(2) We say that $\Lambda=\left[\lambda_{1}, \lambda_{2}\right]$ is real rhombic if there exist generators such that $\lambda_{2}=\overline{\lambda_{1}}$. A lattice similar to a real rhombic lattice is rhombic.

(3) A lattice $\Lambda$ is square if $i \Lambda=\Lambda$. (Equivalently, $\Lambda$ is square if it is similar to a lattice generated by $[\lambda, \lambda i]$, for some $\lambda>0$.)

(4) A lattice $\Lambda$ is triangular if $\Lambda=e^{2 \pi i / 3} \Lambda$ (in which case a period parallelogram can be made from two equilateral triangles).

In each of cases (1)-(3), the period parallelogram with vertices $0, \lambda_{1}, \lambda_{2}$, and $\lambda_{1}+\lambda_{2}$ can be chosen to be rectangular, rhombic, or square respectively.

Lemma 3.1. A lattice is triangular if and only if it has generators of equal length and forming an angle of $2 \pi / 3$ with each other. Every triangular lattice is given by $\Lambda=\left[\lambda e^{\pi i / 3}, \lambda e^{-\pi i / 3}\right]$ for some $\lambda \in \mathbb{C}$.

It was shown in [9] that for one shape, say a square, which corresponds to the point $i \in B$, there are many types of dynamical behavior and many different Julia 
sets possible. Therefore we fix a point in $B$, and to that point we associate an entire parameter plane of Weierstrass elliptic $\wp$ functions (not all distinct, see Section 6).

In $B$, the rectangular lattices correspond to the vertical ray:

$$
V=\{\operatorname{Re}(\tau)=0, \operatorname{Im}(\tau) \geq 1\},
$$

and the rhombic lattices correspond to

$$
L=\left(\{|\tau|=1\} \cup\left\{\operatorname{Re}(\tau)=-\frac{1}{2}\right\}\right) \cap B .
$$

We relate this setting to the examples studied in [9, all of which are real lattices.

Definition 3.3. A meromorphic function $f: \mathbb{C} \rightarrow \mathbb{C}_{\infty}$ is real if $f(\bar{z})=\overline{f(z)}$ for all $z \in \mathbb{C}($ where $\bar{\infty}=\infty)$. A lattice $\Lambda$ is real if $\bar{\Lambda}=\Lambda$.

Theorem 3.2 ([11]). A lattice $\Lambda$ is real if and only if it is real rectangular or real rhombic.

We remark that the real lattices can only occur at rectangular and rhombic points of $B$; that is, along $L$ and $V$.

\section{Weierstrass $\wp$ INVARIANTS AND THE POSTCRITICAL SET}

An elliptic function is a meromorphic function in $\mathbb{C}$ which is periodic with respect to a lattice $\Lambda$. For any $z \in \mathbb{C}$ and any lattice $\Lambda$, the Weierstrass elliptic function is defined by

$$
\wp_{\Lambda}(z)=\frac{1}{z^{2}}+\sum_{w \in \Lambda \backslash\{0\}}\left(\frac{1}{(z-w)^{2}}-\frac{1}{w^{2}}\right) .
$$

Replacing every $z$ by $-z$ in the definition we see that $\wp_{\Lambda}$ is an even function. It is well known that $\wp_{\Lambda}$ is meromorphic, is periodic with respect to $\Lambda$, and has order 2 .

The derivative of the Weierstrass elliptic function is also an elliptic function which is periodic with respect to $\Lambda$ defined by

$$
\wp_{\Lambda}^{\prime}(z)=-2 \sum_{w \in \Lambda} \frac{1}{(z-w)^{3}} .
$$

The Weierstrass elliptic function and its derivative are related by the differential equation

$$
\wp_{\Lambda}^{\prime}(z)^{2}=4 \wp_{\Lambda}(z)^{3}-g_{2} \wp_{\Lambda}(z)-g_{3},
$$

where $g_{2}(\Lambda)=60 \sum_{w \in \Lambda \backslash\{0\}} w^{-4}$ and $g_{3}(\Lambda)=140 \sum_{w \in \Lambda \backslash\{0\}} w^{-6}$.

The numbers $g_{2}(\Lambda)$ and $g_{3}(\Lambda)$ are invariants of the lattice $\Lambda$ in the following sense: if $g_{2}(\Lambda)=g_{2}\left(\Lambda^{\prime}\right)$ and $g_{3}(\Lambda)=g_{3}\left(\Lambda^{\prime}\right)$, then $\Lambda=\Lambda^{\prime}$. Furthermore, given any $g_{2}$ and $g_{3}$ such that $g_{2}^{3}-27 g_{3}^{2} \neq 0$ there exists a lattice $\Lambda$ having $g_{2}=g_{2}(\Lambda)$ and $g_{3}=g_{3}(\Lambda)$ as its invariants 7 .

Theorem 4.1 ([7]). For $\Lambda_{\tau}=[1, \tau]$, the functions $g_{i}(\tau)=g_{i}\left(\Lambda_{\tau}\right), i=2,3$, are analytic functions of $\tau$ in the open upper half plane $\operatorname{Im}(\tau)>0$.

We have the following homogeneity in the invariants $g_{2}$ and $g_{3}$.

Lemma 4.2. For lattices $\Lambda$ and $\Lambda^{\prime}$,

$$
\Lambda^{\prime}=k \Lambda \Leftrightarrow g_{2}\left(\Lambda^{\prime}\right)=k^{-4} g_{2}(\Lambda) \quad \text { and } \quad g_{3}\left(\Lambda^{\prime}\right)=k^{-6} g_{3}(\Lambda) ;
$$


equivalently, assuming $g_{3}(\Lambda) \neq 0$, we have

$$
\Lambda^{\prime}=k \Lambda \Leftrightarrow \frac{\left(g_{2}(\Lambda)\right)^{3}}{27\left(g_{3}(\Lambda)\right)^{2}}=\frac{\left(g_{2}\left(\Lambda^{\prime}\right)\right)^{3}}{27\left(g_{3}\left(\Lambda^{\prime}\right)\right)^{2}} .
$$

Proof. Consider the lattice $\Lambda=[1, \tau]$. The function

$$
j(\tau)=\frac{\left(g_{2}(\tau)\right)^{3}}{27\left(g_{3}(\tau)\right)^{2}}=\frac{\left(g_{2}(\Lambda)\right)^{3}}{27\left(g_{3}(\Lambda)\right)^{2}}
$$

is meromorphic in the upper half plane and attains each value in $\mathbb{C} \neq 1$ at exactly one point in each fundamental region (we assign it to have the value $\infty$ if $g_{3}=0$ ) 7. Therefore, it follows that

$$
\frac{\left(g_{2}(\Lambda)\right)^{3}}{27\left(g_{3}(\Lambda)\right)^{2}}=\frac{\left(g_{2}\left(\Lambda^{\prime}\right)\right)^{3}}{27\left(g_{3}\left(\Lambda^{\prime}\right)\right)^{2}},
$$

if and only if the lattices lie in the same $\Gamma$ orbit, or equivalently, correspond to similar lattices.

The rest of the proof follows immediately from the definition of the invariants.

Theorem 4.3 ([1] $)$. The following are equivalent:

(1) $\wp_{\Lambda}(\bar{z})=\overline{\wp_{\Lambda}(z)}$;

(2) $\Lambda$ is a real lattice;

(3) $g_{2}, g_{3} \in \mathbb{R}$.

For any lattice $\Lambda$, the Weierstrass elliptic function and its derivative satisfy the following properties: for $k \in \mathbb{C} \backslash\{0\}$,

$$
\begin{aligned}
& \wp_{k \Lambda}(k u)=\frac{1}{k^{2}} \wp_{\Lambda}(u), \quad\left(\text { homogeneity of } \wp_{\Lambda}\right), \\
& \wp_{k \Lambda}^{\prime}(k u)=\frac{1}{k^{3}} \wp_{\Lambda}^{\prime}(u), \quad\left(\text { homogeneity of } \wp_{\Lambda}^{\prime}\right) .
\end{aligned}
$$

Verification of the homogeneity properties can be seen by substitution into the series definitions.

We can determine the critical values of the Weierstrass elliptic function on an arbitrary lattice $\Lambda=\left[\lambda_{1}, \lambda_{2}\right]$. For $j=1,2$, notice that $\wp_{\Lambda}\left(\lambda_{j}-z\right)=\wp_{\Lambda}(z)$ for all $z$. Taking derivatives of both sides we obtain $-\wp_{\Lambda}^{\prime}\left(\lambda_{j}-z\right)=\wp_{\Lambda}^{\prime}(z)$. Substituting $z=\lambda_{1} / 2, \lambda_{2} / 2$, or $\left(\lambda_{1}+\lambda_{2}\right) / 2$, we see that $\wp_{\Lambda}^{\prime}(z)=0$ at these values. We use the notation

$$
e_{1}=\wp_{\Lambda}\left(\frac{\lambda_{1}}{2}\right), \quad e_{2}=\wp_{\Lambda}\left(\frac{\lambda_{2}}{2}\right), \quad e_{3}=\wp_{\Lambda}\left(\frac{\lambda_{1}+\lambda_{2}}{2}\right)
$$

to denote the critical values. Since $e_{1}, e_{2}, e_{3}$ are the distinct zeros of Equation (2), we also write

$$
\wp_{\Lambda}^{\prime}(z)^{2}=4\left(\wp_{\Lambda}(z)-e_{1}\right)\left(\wp_{\Lambda}(z)-e_{2}\right)\left(\wp_{\Lambda}(z)-e_{3}\right) .
$$

Equating like terms in Equations (2) and (4), we obtain

$$
e_{1}+e_{2}+e_{3}=0, \quad e_{1} e_{3}+e_{2} e_{3}+e_{1} e_{2}=\frac{-g_{2}}{4}, \quad e_{1} e_{2} e_{3}=\frac{g_{3}}{4} .
$$

There are also formulas for the quarter period values of the Weierstrass elliptic function; we mention one which is of use in constructing an example in Section 10. Let

$$
d_{i}^{2}=\left(e_{i}-e_{j}\right)\left(e_{i}-e_{k}\right)=3 e_{i}^{2}-g_{2} / 4
$$


where we choose the square root so that

$$
\wp_{\Lambda}\left(\lambda_{i} / 4\right)=e_{i}+d_{i} .
$$

Proposition 4.1 ([7]). If $\Lambda$ is a real rectangular lattice, then $e_{1}+d_{1}>e_{1}>e_{3}>$ $e_{1}-d_{1}>e_{2}$.

The lattice shape relates to the properties and dynamics of the corresponding Weierstrass elliptic function as discussed in 9 for the real case. We denote by $p(x)=4 x^{3}-g_{2} x-g_{3}$, the polynomial associated with $\Lambda$, and define $\triangle=g_{2}^{3}-27 g_{3}^{2}$ to be its discriminant. The next several results relate the lattice shapes with the critical values of $\wp_{\Lambda}$.

Proposition 4.2 ([7]). $\quad$ (1) If $\Lambda$ is square, then $g_{3}=0$; in this case the roots of $p$ are $0, \pm \sqrt{g_{2}} / 2$, so $e_{3}=0, e_{1}=\sqrt{g_{2}} / 2$, and $e_{2}=-e_{1}$.

(2) If $\Lambda$ is triangular, then $g_{2}=0$; in this case the roots of $p$ are the cube roots of $g_{3} / 4$, none of which is zero. Therefore $e_{1}, e_{2}, e_{3}$ all have the same modulus and are cube roots of $g_{3} / 4$. Furthermore, $e_{i}$ is real for some $i=1,2,3$ if and only if $g_{3}$ is real and $\Lambda$ is a real lattice.

The symmetry of these lattices is reflected in the behavior of the postcritical sets; we proved the following result in 9] (Prop. 4.8, (3) and (4)) for real lattices, but the proof remains the same in the generality stated here (and follows from Proposition 4.2).

Proposition 4.3. $\quad$ (1) If $\Lambda$ is a square lattice, then $P\left(\wp_{\Lambda}\right)$ includes $\infty$, and $e_{3} \in J\left(\wp_{\Lambda}\right)$. Furthermore, since $e_{1}=-e_{2}$, and $\wp_{\Lambda}$ is even, $P\left(\wp_{\Lambda}\right)=$ $\bigcup_{n \geq 0} \wp_{\Lambda}^{n}\left(e_{1}\right) \cup\left\{e_{2}, 0, \infty\right\}$ (so is completely determined by the forward orbit of $e_{1}$ ).

(2) If $\Lambda$ is triangular, then $P\left(\wp_{\Lambda}\right)$ is contained in three forward invariant sets: one set $\alpha=\overline{\bigcup_{n \geq 0} \wp_{\Lambda}^{n}\left(e_{1}\right)}$, and the sets $e^{2 \pi / 3} \alpha$ and $e^{4 \pi / 3} \alpha$. (These sets are not necessarily disjoint.)

\section{Properties of the Fatou set}

The algebraic and analytic properties of the Weierstrass elliptic function manifest themselves in the large amount of symmetry found in each Julia set arising from an elliptic $\wp$ function. The proof of Theorem 5.1 is given in [9].

Theorem 5.1 (9]). If $\Lambda=\left[\lambda_{1}, \lambda_{2}\right]$ is any lattice, then we have the following:

(1) $J\left(\wp_{\Lambda}\right)+\Lambda=J\left(\wp_{\Lambda}\right)$ and $F\left(\wp_{\Lambda}\right)+\Lambda=F\left(\wp_{\Lambda}\right)$.

(2) $(-1) J\left(\wp_{\Lambda}\right)=J\left(\wp_{\Lambda}\right)$ and $(-1) F\left(\wp_{\Lambda}\right)=F\left(\wp_{\Lambda}\right)$.

(3) $\overline{J\left(\wp_{\Lambda}\right)}=J\left(\wp_{\bar{\Lambda}}\right)$ and $\overline{F\left(\wp_{\Lambda}\right)}=F\left(\wp_{\bar{\Lambda}}\right)$.

(4) If $\Lambda$ is square, then $e^{\pi i / 2} J\left(\wp_{\Lambda}\right)=J\left(\wp_{\Lambda}\right)$ and $e^{\pi i / 2} F\left(\wp_{\Lambda}\right)=F\left(\wp_{\Lambda}\right)$.

(5) If $\Lambda$ is triangular, then $e^{2 \pi i / 3} J\left(\wp_{\Lambda}\right)=J\left(\wp_{\Lambda}\right)$ and $e^{2 \pi i / 3} F\left(\wp_{\Lambda}\right)=F\left(\wp_{\Lambda}\right)$. Moreover, $e^{2 \pi i / 3} \wp_{\Lambda}(z)=\wp_{\Lambda}\left(e^{2 \pi i / 3} z\right)$ for all $z \in \mathbb{C} \backslash \Lambda$.

In addition to a basic Julia set pattern repeating on each fundamental period, we also see symmetry within the period parallelogram.

Proposition 5.1. For the lattice $\Lambda=\left[\lambda_{1}, \lambda_{2}\right], J\left(\wp_{\Lambda}\right)$ and $F\left(\wp_{\Lambda}\right)$ are symmetric with respect to any critical point $\lambda_{1} / 2+\Lambda, \lambda_{2} / 2+\Lambda$, and $\left(\lambda_{1}+\lambda_{2}\right) / 2+\Lambda$. That is, if $c$ is any critical point of $\wp_{\Lambda}$, then $c+z \in J\left(\wp_{\Lambda}\right)$ if and only if $c-z \in J\left(\wp_{\Lambda}\right)$. 
In particular, if $F_{O}$ is any component of $F\left(\wp_{\Lambda}\right)$ that contains a critical point $c$, then $F_{o}$ is symmetric with respect to $c$.

Proof. We have $\wp_{\Lambda}(z)=\wp_{\Lambda}(u)$ if $u \equiv-z \bmod \Lambda$. We first consider $c=\lambda / 2$, where $\lambda$ is one of $\lambda_{1}, \lambda_{2}$, or $\left(\lambda_{1}+\lambda_{2}\right)$. We note also that $\lambda-c=c$. Then $\wp_{\Lambda}(c+z)=\wp_{\Lambda}(-c-z+\lambda)=\wp_{\Lambda}(c-z)$ as claimed. If $c_{m, n}=c+m \lambda_{1}+n \lambda_{2}$, then $\wp_{\Lambda}\left(c_{m, n}+z\right)=\wp_{\Lambda}(c+z)=\wp_{\Lambda}(c-z)=\wp_{\Lambda}\left(c_{m, n}-z\right)$.

5.1. The torus $\mathbb{C} / \Lambda$. In light of the symmetry results of Theorem 5.1 we can view the Julia set on the torus $\mathbb{C} / \Lambda$, and study its topology in that setting as well as in the plane or on the sphere. Let $\pi\left(=\pi_{\Lambda}\right)$ denote the quotient map from the plane to the torus. Each point $z \in \mathbb{C}$ determines a coset $[z]=z+\Lambda \in \mathbb{C} / \Lambda$ under $\pi$ (also called a residue class $(z \bmod \Lambda))$. Since, except at lattice points,

$$
u \in[z] \Rightarrow \wp_{\Lambda}(u)=\wp_{\Lambda}(z) \in\left[\wp_{\Lambda}(z)\right],
$$

the map $\wp_{\Lambda}$ determines an analytic map on the torus minus the point at [0] (the image of the lattice points) onto itself, but does not extend to a holomorphic map of the torus. We therefore have a well-defined map $A_{\Lambda}([z])=\pi \circ \wp_{\Lambda} \circ \pi^{-1}[z], z \neq[0]$. This gives a holomorphic map $A_{\Lambda}: \mathbb{C} / \Lambda \backslash\{[0]\} \rightarrow \mathbb{C} / \Lambda$ induced by $\wp_{\Lambda}$ on $\mathbb{C}$; it cannot be extended to the whole torus. The map $A_{\Lambda}$ is countably infinite-to-one at every point on which it is defined; $A_{\Lambda}$ can be iterated infinitely often at the points that never get mapped to [0].

The obvious definitions of Fatou and Julia sets for $A_{\Lambda}$ on $\mathbb{C} / \Lambda$ (using normal families) give sets which agree with the sets $\pi\left(J\left(\wp_{\Lambda}\right)\right)$ and $\pi\left(F\left(\wp_{\Lambda}\right)\right)$.

5.2. Classification of Fatou components. We now turn to a discussion about the possible periodic Fatou components for $\wp_{\Lambda}$.

Lemma $5.2(9])$. For any lattice $\Lambda$, the Fatou set of $\wp_{\Lambda}$ has no wandering domains and no Baker domains.

Proof. $\wp_{\Lambda}$ has only three critical values. Since $\wp_{\Lambda}$ is doubly periodic, it cannot have any asymptotic values. Therefore it is a Class $\mathrm{S}$ function and has no wandering domains [3] or Baker domains 8 .

Furthermore, we have a bound on the number of Fatou cycles that can occur for a Weierstrass elliptic map.

Theorem 5.3. For any lattice $\Lambda$, at most three different types of periodic Fatou cycles can occur for $\wp_{\Lambda}$.

Proof. The map $\wp_{\Lambda}$ has three distinct critical values with at most three distinct orbits.

The following theorem strengthens a result in [9] (Lemma 6.3) which was proved under additional hypotheses.

Theorem 5.4. For any lattice $\Lambda, \wp_{\Lambda}$ has no cycle of Herman rings.

Proof. Suppose that $\wp_{\Lambda}$ has a cycle of Herman rings $\left\{U_{0}, U_{1}, \ldots, U_{p-1}\right\}$ of period $p$. Then for any $i=0,1, \ldots, p-1, \wp_{\Lambda}^{p}: U_{i} \rightarrow U_{i}$ is conjugate to an irrational rotation of the annulus and thus has degree one. The preimages under this conjugacy of the circles $|\eta|=r, 1<r<R$ foliate the disks with $\wp_{\Lambda}^{p}$ forward invariant leaves on which $\wp_{\Lambda}^{p}$ is injective. Let $\gamma$ be a $\wp_{\Lambda}^{p}$ invariant leaf of $U_{i}$, and let $B_{\gamma}$ denote the 
bounded component of the complement of $\gamma$. Since $U_{i}$ is multiply connected, we know that $B_{\gamma}$ contains a prepole. Thus, there is a smallest nonnegative number $n$ such that $\wp_{\Lambda}^{n}(\gamma)$ contains a lattice point $\omega$ in $B_{\wp_{\Lambda}^{n}(\gamma)}$. Let $U_{j}$ denote the Herman ring $\wp_{\Lambda}^{n}\left(U_{i}\right)$.

Since $U_{j}$ is homeomorphic to an annulus with the lattice point $\omega$ in $B_{\wp_{\Lambda}^{n}(\gamma)}$, Theorem 5.1 (1) and (2) imply that $-U_{j}+2 \omega$ is a Fatou component such that $\omega$ is in $-B_{\wp_{\Lambda}^{n}(\gamma)}+2 \omega$. Since the topology is identical in every fundamental region, we assume by translating the entire setup by $-\omega$, that $\omega=0$. Therefore both $U_{j}$ and $-U_{j}$ are annuli containing simple closed loops $\gamma$ and $-\gamma$ respectively, and each has 0 in its bounded complement. If we show that there is one point $z \in U_{j} \cap-U_{j}$, then by symmetry of the Fatou set it follows that $U_{j}$ is symmetric with respect to 0 and $U_{j}=-U_{j}$.

For each point $z \in \gamma$, we assign coordinates $z=(r, \theta)$. Since $\gamma$ encloses the origin, each value of $\theta \in[0,2 \pi)$ is attained as a coordinate for exactly one point of $\gamma$. We set $z_{o}=\left(r_{o}, 0\right) \in \gamma$, then consider $z_{1}=\left(r_{1}, 0\right) \in-\gamma$. If $r_{1}=r_{o}$, then we are done since $z_{o}=z_{1}$, so we assume that $r_{1}<r_{o}$. We have $w_{o}=\left(r_{o}, \pi\right) \in-\gamma$ and $w_{1}=\left(r_{1}, \pi\right) \in \gamma$. Considering now the continuous function $r(\theta)=r_{+}-r_{-}$, where $r_{+}$is the radial coordinate for $\gamma$ at $\theta$, and $r_{-}$is the radial coordinate for $-\gamma$ at $\theta$, we have that $r(0)>0$ and $r(\pi)<0$; by the intermediate value theorem, $r$ must be 0 at some angle $\theta_{o} \in(0, \pi)$, so the point $z=\left(r\left(\theta_{o}\right), \theta_{o}\right)$ lies in $\gamma$ and $-\gamma$.

Then translating back to the original $\omega$, we have that if $z \in U_{j}$, then $-z+2 \omega \in$ $U_{j}$. However, since $\wp_{\Lambda}(z)=\wp_{\Lambda}(-z+2 \omega), \wp_{\Lambda}^{p}$ cannot be degree one on $U_{j}$ which is a contradiction.

The periodicity with respect to $\Lambda$ gives rise to different possibilities for connectivity of a component when the Fatou and Julia sets are projected to the torus.

Definition 5.1. A forward invariant Fatou component $F_{o}$ of the map $\wp_{\Lambda}$ is called a toral band if $F_{o}$ contains an open subset $U$ which is simply connected in $\mathbb{C}$, but $\pi_{\Lambda}(U)$ is not simply connected on $\mathbb{C} / \Lambda$. In other words, $U$ projects to a set in the torus containing a homotopically nontrivial loop.

Proposition 5.2. For any lattice $\Lambda, \wp_{\Lambda}$ has a toral band if and only if there is a component of the Fatou set which is not completely contained in one fundamental region of $\mathbb{C} / \Lambda$.

Proof. Suppose that $\Lambda=\left[\lambda_{1}, \lambda_{2}\right]$. By Definition [3.1] the map $\wp_{\Lambda}$ has a component $F_{o} \subset F\left(\wp_{\Lambda}\right)$ with the property that $F_{o}$ is not contained in one fundamental region if and only if there exists a point $a \in F_{o}$ and a lattice point $\lambda=m \lambda_{1}+n \lambda_{2}$ (for some integers $m, n$, not both 0 ) such that $a+\lambda \in F_{o}$. Since $F_{o}$ is open and connected, it is path connected so there exists a path $p$ in $F_{o}$ such that $p(0)=a$ and $p(1)=a+\lambda$. Then $p$ is the lifting of a loop $q$ at $[a]$ on $\mathbb{C} / \Lambda$; in particular, the homotopy class of the loop $q$ is the group element $\lambda \in \Lambda$ (since $\Lambda$ is isomorphic to the fundamental group of $\mathbb{C} / \Lambda)$. Then the open set $\pi\left(F_{o}\right)$ contains the nontrivial loop $q$, and this is equivalent to the existence of a toral band for $\wp_{\Lambda}$.

We prove in Section[10 that toral bands occur for some rectangular lattice shapes. For some particular lattice shapes, there are additional restrictions on the types of Fatou components that can occur. 
Proposition 5.3. For any triangular lattice $\Lambda=\left[\lambda, e^{2 \pi i / 3} \lambda\right]$, one of the following must occur:

(1) $J\left(\wp_{\Lambda}\right)=\mathbb{C}_{\infty}$.

(2) For some period $n$ and some multiplier $\beta, 0 \leq \beta \leq 1$ there exist exactly three (super)attracting periodic cycles or parabolic periodic cycles in the Fatou set of period $n$ with multiplier $\beta$.

(3) There exists exactly one (super)attracting periodic cycle or parabolic periodic cycle in the Fatou set which contains all three critical values.

(4) The only Fatou cycles are Siegel disks.

In any case, no two critical values lie in the same component of the Fatou set.

Proof. If the Julia set is not the entire sphere, then there exists a cycle of Fatou components. By Lemma 5.2 and Theorem [5.4 $\wp_{\Lambda}$ has no Herman rings or Baker domains. If $\wp_{\Lambda}$ has a cycle of attracting basins or parabolic domains, then the cycle must contain a critical value $e_{i}$. Proposition 4.2 and Theorem 5.1 imply that all critical values must lie in the Fatou set and thus converge to attracting or parabolic cycles. Theorem 5.1 implies that the critical values either converge to three periodic cycles or they all converge to one periodic cycle. If there are three periodic cycles, then the homogeneity property of the derivative implies that they must have the same classification. If there is a cycle of Siegel disks, then the boundary of the cycle must be contained in the closure of the forward orbit of the critical points, and thus there must be at least one critical value $e_{i}$ in the Julia set. Proposition 4.2 and the homogeneity property imply that all critical values must lie in the Julia set, and thus the only possible Fatou cycles are Siegel disks.

To finish the proof, we show that if there exists an attracting or parabolic cycle, then $e_{1}, e_{2}$, and $e_{3}$ lie in pairwise disjoint components of $F\left(\wp_{\Lambda}\right)$. Let $\left\{U_{1}, U_{2}, \ldots, U_{n}\right\}$ denote a forward invariant cycle of Fatou components corresponding to such a cycle $\left\{p_{1}, p_{2}, \ldots, p_{n}\right\}$. Some component, $U_{j}$, contains a critical value, say $e_{1}$. Then $\lim _{k \rightarrow \infty} \wp_{\Lambda}^{k n}\left(e_{1}\right)=p_{j}$, and by Theorem [5.1, $\lim _{k \rightarrow \infty} \wp_{\Lambda}^{k n}\left(e_{2}\right)=e^{2 \pi i / 3} p_{j}$ and $\lim _{k \rightarrow \infty} \wp_{\Lambda}^{k n}\left(e_{3}\right)=e^{4 \pi i / 3} p_{j}$. Therefore, if $e_{2}$ lies in $U_{j}$, then $p_{j}=e^{2 \pi i / 3} p_{j}$, so $p_{j}=0$, which is a pole and therefore contradicts the hypothesis. The same argument shows that no pair of critical values lies in one component of the cycle.

Remark. In a later section we prove that each of the possibilities (1)-(3) listed in Proposition 5.3 occurs for some triangular lattice.

Proposition 5.4. For any square lattice $\Lambda=[\lambda, \lambda i]$, one of the following must occur:

(1) $J\left(\wp_{\Lambda}\right)=\mathbb{C}_{\infty}$.

(2) There exists exactly one (super)attracting or parabolic periodic cycle of the Fatou set.

(3) The only periodic Fatou cycles are Siegel disks.

Proof. Proposition 4.2 implies that $e_{3}$ is in the Julia set, and the forward orbits of $e_{1}$ and $e_{2}$ coincide. Thus there is only one critical orbit that could be associated with components of the Fatou set. By Lemma 5.2 and Theorem $5.4 \wp_{\Lambda}$ has no Herman rings or Baker domains. 


\section{The PARAMETER Planes FOR A LATTICE SHAPE}

We study the parameter space for the elliptic functions $\wp_{\Lambda}$ using the theory of holomorphic families as introduced by Mañé, Sad, and Sullivan [16], refined by McMullen [20], and generalized to the setting of meromorphic maps with finite singular set by Keen and Kotus [12. We give an overview of the main results here.

Definition 6.1. (1) A holomorphic family of meromorphic maps $f_{\lambda}(z)$ over a complex manifold $M$ is a holomorphic map $M \times \mathbb{C}_{\infty} \rightarrow \mathbb{C}_{\infty}$, given by $(\lambda, z) \mapsto f_{\lambda}(z)$

(2) $M^{\text {top }} \subset M$ is the set of points $\lambda$ that have a neighborhood $U$ with the property that $\omega \in U$ implies there is a homeomorphism $\phi: \mathbb{C}_{\infty} \rightarrow \mathbb{C}_{\infty}$ such that $f_{\omega}=\phi^{-1} \circ f_{\lambda} \circ \phi$.

(3) The set $M^{q c} \subset M$ is defined similarly except that $\phi$ must be quasiconformal.

(4) Suppose that $f_{\lambda}$ has a finite singular set for every $\lambda \in M$. Let $M_{o} \subset M$ be the set of parameters such that the number of singular values of $f_{\lambda}$ is locally constant. For $\lambda \in M_{o}$, the singular values can be locally labelled by holomorphic functions $s_{1}(\lambda), s_{2}(\lambda), \ldots, s_{n}(\lambda)$. A singular orbit relation is a set of integers $(i, j, m, n)$ with $m, n \geq 0$ such that $f^{m}\left(s_{i}(\lambda)\right)=f^{n}\left(s_{j}(\lambda)\right)$. The set $M^{\text {post }} \subset M_{o}$ of postsingularly stable parameters consists of all $\lambda$ such that the set of singular orbit relations is locally constant.

(5) A holomorphic motion of a set $J \subset \mathbb{C}_{\infty}$ over a connected complex manifold with basepoint $\left(M, \lambda_{o}\right)$ is a map $\phi: M \times J \rightarrow \mathbb{C}_{\infty}$ given by $(\lambda, z) \mapsto \phi_{\lambda}(z)$ satisfying:

- for each fixed $z \in J, \phi_{\lambda}(z)$ is holomorphic in $\lambda$;

- for each fixed $\lambda, \phi_{\lambda}(z)$ is an injective function of $z$;

- $\phi_{\lambda_{o}}(z)=z$; i.e., it is the identity function at the basepoint.

(6) A holomorphic motion over $\left(M, \lambda_{o}\right)$ respects the dynamics if

$$
\phi_{\lambda}\left(f_{\lambda_{o}}(z)\right)=f_{\lambda}\left(\phi_{\lambda}(z)\right),
$$

whenever $z$ and $f_{\lambda_{o}}(z)$ both belong to $J$.

(7) The set $M^{\text {stab }} \subset M$ denotes the $J$-stable set of parameters such that the Julia set moves by a holomorphic motion respecting the dynamics.

The next theorem was proved in [12, following the methods of [20].

Theorem 6.1. For any holomorphic family of meromorphic maps with finite singular set defined over the complex manifold $M$ :

(1) The topologically stable parameters are open and dense.

(2) $M^{q c}=M^{\text {post }}=M^{\text {top }}$.

(3) $M^{\text {stab }}$ is the set of parameters for which the total number of attracting and superattracting cycles of $f_{\lambda}$ is constant in a neighborhood of $\lambda$.

(4) $M^{\text {stab }}$ is open and dense in $M$.

As is frequently the case, properties possessed by the Julia sets of a holomorphic family of maps are reflected in parameter space; indeed intricate symmetry and periodicity are seen in every parameter space we view in the setting of Weierstrass $\wp$ functions. Additionally, Theorem 6.1 shows that one expects to see Mandelbrotlike sets, and they occur here too.

One way to study parameter space for the Weierstrass elliptic $\wp$ function is to fix a lattice shape $\tau \in B$ (see Equation (1)) and then look at the space of all lattices of that shape determined by the value $\tau$. 


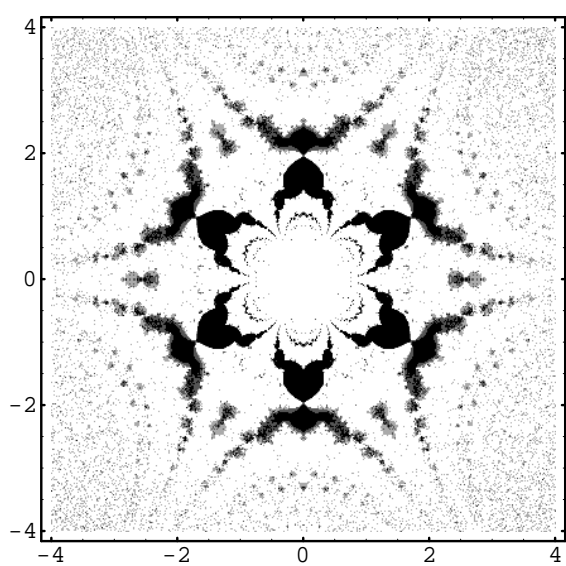

FiguRE 2. $k$-space for $\Lambda=[1,2 i]$

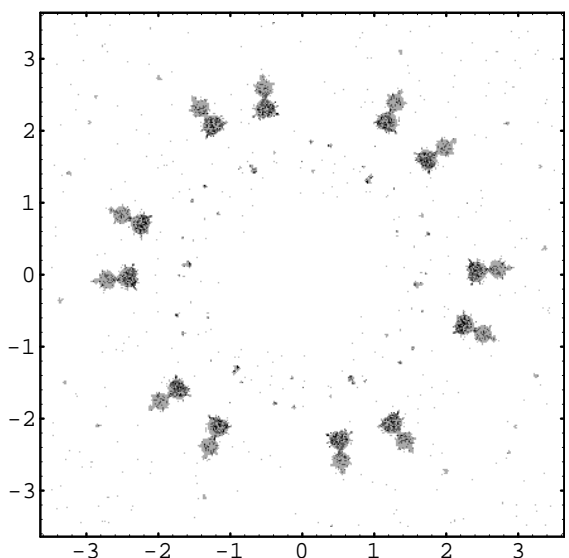

FiguRE 3. $k$-space for $\Lambda=[1, .25+i]$

Definition 6.2. For a given lattice $\Lambda=[1, \tau]$, with $\tau \in B$, we define $k$-space to be the set of points $k \in \mathbb{C} \backslash\{0\}$ which represent the lattice $k \Lambda$ and therefore the function $\wp_{k \Lambda}$.

If we fix a value $\tau$ and the corresponding lattice $\Lambda=[1, \tau]$, then the map:

$$
F_{\tau}: \mathbb{C} \backslash\{0\} \times \mathbb{C} \rightarrow \mathbb{C}_{\infty}
$$

given by $F_{\tau}(k, z)=\wp_{k \Lambda}(z)$ is holomorphic in $k$ and meromorphic in $z$. This defines a holomorphic family of meromorphic functions; we say that the holomorphic family of meromorphic maps parametrized over a complex manifold $M$ is reduced if for all $k \neq k^{\prime}$ in $M, \wp_{k \Lambda}$ and $\wp_{k^{\prime} \Lambda}$ are not conformally conjugate. In the current setting, $k$ space is far from reduced; the next results distinguish the symmetries of parameter space stemming from conjugate copies from the symmetries that occur for other reasons.

6.1. The reduction of $k$-space. We use the computer software Mathematica 17 . to visualize $k$-space; several examples are shown here. The algorithm checks how many critical values tend to attracting periodic orbits under $\wp_{k \Lambda}^{n}$ as $n \rightarrow \infty$. The point $k$ is colored white if none of the critical orbits converge to periodic orbits, light grey if one does, dark grey if two do, and black if all three converge to periodic cycles. Figure 2 shows the $k$-space for the rectangular lattice shape based at $\tau=2 i$. At the parameter value $k=1$, we see the information about the number of critical values heading to attracting periodic orbits for the Weierstrass elliptic function on the lattice $\Lambda=[1,2 i]$. Figure 3 shows a typical $k$-space.

In every $k$-space, six-fold symmetry is visible; we prove that this is indeed the case. Since $k \Lambda=-k \Lambda$ for any $k$, we have symmetry with respect to rotation by $\pi$ in $k$-space. We describe the additional rotational symmetry in $k$-space in the next result.

Theorem 6.2. If $\Gamma$ is any lattice and $\Omega=e^{\pi i / 3} \Gamma$, then $\wp_{\Gamma}$ and $\wp_{\Omega}$ are conformally conjugate via the map $\psi(z)=-e^{\pi i / 3} z=e^{4 \pi i / 3} z$. In this case, $g_{2}(\Omega)=e^{2 \pi i / 3} g_{2}(\Gamma)$ and $g_{3}(\Omega)=g_{3}(\Gamma)$. Moreover, if $\left\{z_{0}, z_{1}, \ldots, z_{m-1}\right\}$ is a cycle with multiplier $\beta$ for $\wp_{\Gamma}$, then there is a corresponding cycle of period $m$ with multiplier $(-1)^{m} \beta$ for $\wp_{\Omega}$. 
Proof. The proof is an application of the homogeneity property (Equation (3)):

$$
\begin{gathered}
\wp_{\Omega}\left(-e^{\pi i / 3} z\right)=\wp_{\Omega}\left(e^{\pi i / 3} z\right)=\frac{1}{e^{2 \pi i / 3} \wp_{\Gamma}(z)} \\
=e^{4 \pi i / 3} \wp_{\Gamma}(z)=-e^{\pi i / 3} \wp_{\Gamma}(z) .
\end{gathered}
$$

The homogeneity property of the derivative implies

$$
\wp_{\Omega}^{\prime}\left(e^{\pi i / 3} z\right)=\frac{1}{e^{3 \pi i / 3}} \wp_{\Gamma}^{\prime}(z)=-\wp_{\Gamma}^{\prime}(z)
$$

(so the types of cycles carry over).

As seen in Lemma 4.2,

$$
g_{2}(\Omega)=\frac{1}{\left(-e^{\pi i / 3}\right)^{4}} g_{2}(\Gamma)=e^{2 \pi i / 3} g_{2}(\Gamma) .
$$

Additionally,

$$
g_{3}(\Omega)=\frac{1}{\left(-e^{\pi i / 3}\right)^{6}} g_{3}(\Gamma)=g_{3}(\Gamma)
$$

We now describe the possible conformal conjugacies that can occur between two maps $\wp_{\Lambda}$ and $\wp_{\Gamma}$.

Lemma 6.3. If $\wp_{\Gamma}$ is conformally conjugate to $\wp_{\Lambda}$ via a Möbius map $\phi$, then $\phi(z)=a z$ and $\phi(\Gamma)=\Lambda$.

Proof. Assume that $\phi(z)=\frac{a z+b}{c z+d}$ and $\phi \circ \wp_{\Gamma}(z)=\wp_{\Lambda} \circ \phi(z)$ for all $z \in \mathbb{C}$. Then $\phi(\infty)=\infty$ (since neither $\wp_{\Lambda}$ nor $\wp_{\Gamma}$ is defined precisely at that point); that is, $\phi$ takes $\mathbb{C}$ onto $\mathbb{C}$. This means that $c=0$ and $\phi$ is affine. Therefore, $\phi(z)=a z+b$ with $a \neq 0$; we have that $\phi(0)=b \in \Lambda$ since it must be a pole of $\wp_{\Lambda}$.

Since critical points must be mapped to critical points under $\phi$, the critical values are mapped to critical values as well. Therefore, if $e_{1, \Gamma}, e_{2, \Gamma}$, and $e_{3, \Gamma}$ denote the critical values of $\wp_{\Gamma}$, then they get mapped under $\phi$ to $e_{1, \Lambda}, e_{2, \Lambda}$, and $e_{3, \Lambda}$ (not necessarily in that order). Furthermore, by Equation (5), we have that

$$
e_{1, \Gamma}+e_{2, \Gamma}+e_{3, \Gamma}=0
$$

and

$$
\begin{aligned}
0 & =e_{1, \Lambda}+e_{2, \Lambda}+e_{3, \Lambda} \\
& =\phi\left(e_{1, \Gamma}\right)+\phi\left(e_{2, \Gamma}\right)+\phi\left(e_{3, \Gamma}\right) \\
& =a\left(e_{1, \Gamma}+e_{2, \Gamma}+e_{3, \Gamma}\right)+3 b=3 b .
\end{aligned}
$$

Therefore $b=0, \phi(z)=a z$, and $\phi$ induces a group isomorphism between $\mathbb{C} / \Gamma$ and $\mathbb{C} / \Lambda$; in particular $a \Gamma=\Lambda$.

In the next result we identify the complex manifold over which we define the reduced holomorphic motion.

Theorem 6.4. For each fixed shape $\tau \neq i, e^{2 \pi i / 3}$, the sector of $k$-space for $\tau$ such that $-\frac{\pi}{6}<\operatorname{Arg}(k) \leq \frac{\pi}{6}$ is a reduced holomorphic family of maps. 
Proof. Let $\Gamma=[1, \tau]$. By our assumption, $\Gamma$ is neither triangular nor square, so we have that $k \Gamma=\Gamma$ implies that $k= \pm 1$. Suppose there are nonzero values $k_{1}$ and $k_{2}$ in the given sector such that $\wp_{k_{1} \Gamma}$ is conformally conjugate to $\wp_{k_{2} \Gamma}$; i.e., $\phi \circ \wp_{k_{1} \Gamma}(z)=\wp_{k_{2} \Gamma} \circ \phi(z)$. We write $\Gamma_{1}=k_{1} \Gamma$ and $\Gamma_{2}=k_{2} \Gamma$. Then, by Lemma 6.3. we have that the conjugacy is implemented by $\phi(z)=a z$ for some $a \in \mathbb{C}$.

However, we know that $\frac{k_{2}}{k_{1}} \Gamma_{1}=\Gamma_{2}$, so $a \Gamma_{1}=\frac{k_{2}}{k_{1}} \Gamma_{1}$, hence $a \frac{k_{1}}{k_{2}} \Gamma_{1}=\Gamma_{1}$. Therefore $a \frac{k_{1}}{k_{2}}= \pm 1$, or

$$
a= \pm \frac{k_{2}}{k_{1}}
$$

If $\phi$ maps the critical values in an order preserving way, then $\phi(z)=\frac{k_{1}^{2}}{k_{2}^{2}} z$; this is impossible unless $k_{1}^{3}= \pm k_{2}^{3}$. Since, by hypothesis, $k_{1}$ and $k_{2}$ lie in the same sector of size $\pi / 3$, this implies that $k_{1}=k_{2}$. Therefore, $\phi$ must permute the postcritical values. We next eliminate the possibility that one of the $e_{i}$ 's is fixed by $\phi$; i.e., suppose that $\phi\left(e_{i} / k_{1}^{2}\right)=e_{i} / k_{2}^{2}$. Then letting $i, j, m$ denote $1,2,3$ in any order, we must have $\phi\left(e_{j} / k_{1}^{2}\right)=e_{j} / k_{2}^{2}=e_{m} / k_{2}^{2}$, unless $e_{i}=0$, so the lattice is square which is a contradiction.

We conclude that the map $\phi$ must permute all of the postcritical values. If $\phi\left(e_{i} / k_{1}^{2}\right)=e_{j} / k_{2}^{2}$, then $a=\frac{e_{j}}{e_{i}} \cdot \frac{k_{1}^{2}}{k_{2}^{2}}$, and $e_{i} / e_{j}=e_{j} / e_{m}=e_{m} / e_{i}, i \neq j \neq m$, and this implies that the lattice is triangular, using Equation (5) to see that $g_{2}=0$. This contradicts the hypothesis that $\tau \neq e^{2 \pi i / 3}$. Therefore, no such map $\phi$ exists.

There is more symmetry in the triangular and square parameter spaces, which we treat separately. First, for triangular lattices, a sector of angle size $\frac{\pi}{3}$ radians in $k$-space is reduced, which is a corollary of the following proposition.

Proposition 6.1. Suppose $\Gamma$ is a triangular lattice such that $\Gamma=\left[k, k e^{2 \pi i / 3}\right]$. If $a=e^{2 \pi i / 9}$, then $a \wp_{\Gamma}(z)=\wp_{a \Gamma}\left(e^{2 \pi i / 3} z\right)$ and $a^{-1} \wp_{\Gamma}(z)=\wp_{a^{-1} \Gamma}\left(e^{-2 \pi i / 3} z\right)$.

Proof. With $a=e^{2 \pi i / 9}$ and $b=e^{2 \pi i / 3} a$, we use the fact that

$$
\Gamma=\frac{a}{b} \Gamma=\frac{b}{a} \Gamma
$$

and the homogeneity property (Equation (3)). Then

$$
a=\frac{1}{b^{2}},
$$

and

$$
\wp_{a \Gamma}(b z)=\wp_{b \Gamma}(b z)=\frac{1}{b^{2}} \wp_{\Gamma}(z)=a \wp_{\Gamma}(z) .
$$

The proof for $a^{-1}$ is the same.

We use this result to show that there is only 6 -fold conjugacy symmetry in triangular $k$-space. It is also used in the next section to show additional symmetries in parameter space which stem from the identity just proved. However, those symmetries do not come from conformal conjugacy as the next result shows.

Corollary 6.5. If $\tau=e^{2 \pi i / 3}$, the sector of $k$-space for $\tau$ such that $-\frac{\pi}{6}<\operatorname{Arg}(k) \leq$ $\frac{\pi}{6}$ is a reduced holomorphic family of maps. 
Proof. We proceed as in Theorem 6.4 arguing as above; if $\phi$ is a conjugating map between $\wp_{k_{1} \Gamma}$ and $\wp_{k_{2} \Gamma}$, it is necessary that

$$
a=e^{ \pm 2 \pi i / 3} \cdot \frac{k_{1}^{2}}{k_{2}^{2}}= \pm \frac{k_{2}}{k_{1}} .
$$

If

$$
a=e^{2 \pi i / 3} \cdot \frac{k_{1}^{2}}{k_{2}^{2}}=\frac{k_{2}}{k_{1}},
$$

then it follows that $\left|k_{1}\right|=\left|k_{2}\right|$, and $k_{2}=e^{2 \pi i / 9} k_{1}$ if both are within the given sector. However, this implies that $a=e^{2 \pi i / 9}$, and by Proposition 6.1, $\phi(z)=a z$ will not implement the conjugacy. (It is easy to see this by evaluating at lattice points.) If

or

$$
a=e^{-2 \pi i / 3} \cdot \frac{k_{1}^{2}}{k_{2}^{2}}=\frac{k_{2}}{k_{1}},
$$

or

$$
a=e^{2 \pi i / 3} \cdot \frac{k_{1}^{2}}{k_{2}^{2}}=-\frac{k_{2}}{k_{1}},
$$

$$
a=e^{-2 \pi i / 3} \cdot \frac{k_{1}^{2}}{k_{2}^{2}}=-\frac{k_{2}}{k_{1}},
$$

then similar arguments show that no map $\phi$ exists.

In the case of square lattices, the corresponding $k$-space exhibits 12 -fold symmetry due to the fact that the reduced sector is smaller than that of all other lattice shapes. We prove that here.

Proposition 6.2. If $\Gamma=[k, i k], k \in \mathbb{C} \backslash\{0\}$, and $\Omega=e^{\pi i / 6} \Gamma$. Then $\psi \circ \wp_{\Gamma}(z)=$ $\wp_{\Omega} \circ \psi(z)$ where $\psi(z)=e^{2 \pi i / 3} z$.

Proof. We use the fact that $i \Gamma=\Gamma$ and the homogeneity property (Equation (3)). We set

Then

$$
\alpha=e^{2 \pi i / 3}=i e^{\pi i / 6}=\left(1 /\left(i e^{\pi i / 6}\right)\right)^{2}=\frac{1}{\alpha^{2}} .
$$

$$
\wp_{\Omega}(\alpha z)=\wp_{i \Omega}(\alpha z)=\frac{1}{\alpha^{2}} \wp_{\Gamma}(z)=\alpha \wp_{\Gamma}(z) .
$$

We have shown in 9] (Theorem 5.2) that for lattices $\Lambda$ and $\bar{\Lambda}$, the map $\phi(z)=$ $\bar{z}$ gives a topological conjugacy between the elliptic functions $\wp_{\Lambda}$ and $\wp_{\bar{\Lambda}}$. The following corollary shows that there are many more topologically conjugate elliptic $\wp$ functions.

Corollary 6.6. For each fixed lattice shape $\tau$, we obtain a reduced holomorphic family of maps $\wp_{k}(z)=\wp_{[k, k \tau]}(z)$ over $M$, with $M \cong \mathbb{C} / \mathbb{Z}$, and $M^{q c} \cap M^{\text {stab }}$ forms an open and dense set in $M$.

In the case of square lattices, we can prove a stronger result.

Proposition 6.3. If $\tau=i$, and $M$ denotes the reduced $k$-space for the square lattices, then the $J$-stable parameters coincide with the quasiconformally stable parameters. 
Proof. By Proposition 4.2(1) we have that for any square lattice, $e_{2}=-e_{1}$, and $e_{3}=$ 0 . Then for any $k \in M$, we always have the postcritical relationship $\wp_{[k, i k]}\left(e_{1}\right)=$ $\wp[k, i k]\left(e_{2}\right)$; furthermore, for every $k, e_{3}$ is a pole and hence is in the Julia set. Since $M^{\text {post }}=M^{\text {top }}$, and since any topologically stable parameter is $J$-stable, we have that $M^{\text {post }} \subset M^{\text {stab }}$. Now suppose that $k \in M^{\text {stab }}$ and that there is a postcritical relation; then writing $\wp_{k}$ for $\wp_{[k, i k]}$, the relation must be of the form:

$$
\wp_{k}^{m}\left(e_{1}(k)\right)=\wp_{k}^{n}\left(e_{1}(k)\right),
$$

for some $m, n \geq 0$. Therefore the forward orbit of $e_{1}$ (and hence $e_{2}$ and the whole postcritical set) is finite. One possibility is that there is no critical point in the finite forward orbit of $e_{1}$, in which case $J\left(\wp_{k}\right)=\mathbb{C}_{\infty}$. In this case there is a holomorphic motion defined on all of $\mathbb{C}_{\infty}$ preserving the dynamics, including the postcritical values and their orbits so that $k \in M^{\text {post }}$.

The only other possibility is that there is a critical point in the finite forward orbit, so that $\wp_{k}$ has a superattracting cycle. However, we claim there is always a $\lambda$ arbitrarily close to $k$ which has an infinite number of points in the forward orbit of $e_{1}(\lambda)$. Therefore there cannot be such a relation on the postcritical set so $k \in M^{\text {post }}$.

The proof of the claim follows from first considering Equations (2) and (4); these show the connection between the derivatives and the postcritical values. These equations applied to Lemma 4.2 and Proposition 4.2 (1) show how changing a side of a square lattice changes a derivative, and Equation (3) gives explicit information about the changes in orbit values as a function of the change in side of the lattice. From these we see that a small change in the side of a square lattice at a superattracting cycle results in an attracting cycle which therefore has an infinite critical orbit.

6.2. Mandelbrot-like sets in $k$-space and $J$-stabililty. The local two-to-one property of every Weierstrass elliptic $\wp_{\Lambda}$ function makes many of them quadraticlike mappings on open sets within a single period parallelogram and away from poles. This places us in a setting where we can apply some results of Douady and Hubbard [6], McMullen [19], and others to explain the Julia sets of Weierstrass $\wp$ functions as well as the appearance of Mandelbrot-like sets in parameter space. We first turn to the notions of hyperbolic and subhyperbolic elliptic maps.

Definition 6.3. We say that an elliptic function is hyperbolic if $J(f)$ is disjoint from the postcritical set $P(f)$. We say $f$ is subhyperbolic if each critical value in the Julia set has a finite forward orbit, while each critical value in the Fatou set is attracted to an attracting cycle.

It follows from the discussion above that $k$-space for square lattices contains no hyperbolic parameters, but there are many subhyperbolic ones. In particular, if we consider square $k$-space and define

$$
\mathcal{H}=\left\{k: \wp_{k} \text { has an attracting periodic orbit }\right\},
$$

then $k \in \mathcal{H}$ is subhyperbolic and the components of $\mathcal{H}$ are components of $M^{\text {stab }}$.

For any lattice shape, the hyperbolic (or in some special cases, subhyperbolic) parameters in $k$-space form a natural subset of $M^{\text {stab }}$, and the bifurcation locus, $M^{b i f}$ is defined to be $M \backslash M^{s t a b}$.

Suppose we start with a lattice $\Lambda_{o}=\left[\lambda_{1}, \lambda_{2}\right]$ such that a superattracting fixed point exists at $\lambda_{1} / 2$. It follows that there exists a neighborhood $U$ of the point $\lambda_{1} / 2$ 
on which $\wp_{\Lambda}$ is conformally conjugate to the map $z \mapsto z^{2}$. Such parameters were shown to exist in [9], and we prove the existence of further examples later in this paper. Since the critical point $\lambda_{1} / 2$, which is a half-lattice point, varies analytically as the pair $\left(g_{2}, g_{3}\right)$ varies, or in a neighborhood of $\Lambda_{o}$, in the parametrizations we consider, we can show there exist polynomial-like mappings nearby of degree 2 using the definition introduced by Douady and Hubbard in [6].

Furthermore, as Figure 9 shows, and McMullen's work predicts [19, in some instances we obtain a Mandelbrot-like set of bifurcations around this parameter, namely the set $M^{b i f}$. We note that in Figure 17we see the Julia set of a Weierstrass elliptic function on a triangular lattice with a period 3 superattracting cycle, where the three cycle is contained within one period parallelogram. In Figure 18, we show the "Douady rabbit"; i.e., the Julia set of a quadratic polynomial of the form $z^{2}+c$ with a superattracting 3 -cycle. Using [6] one easily sees that the two maps are hybrid equivalent, i.e., quasiconformally conjugate as polynomial-like mappings on a neighborhood of their filled Julia sets. However, the proof of the existence of Mandelbrot sets in the bifurcation locus requires the extension of McMullen's results for rational maps to the meromorphic setting which we do not present here.

\section{Parametrizing Real lattices using $\left(g_{2}, g_{3}\right)$-SPace}

Up to now we have discussed parameter spaces associated to a particular lattice shape. Another interesting parameter space is the space of all Weierstrass elliptic functions $\wp_{\Lambda}$ where the invariants $g_{2}(\Lambda)$ and $g_{3}(\Lambda)$ are real. We assume throughout this section that $g_{2}$ and $g_{3}$ are real, and are invariants for a lattice $\Lambda$ and its corresponding elliptic Weierstrass $\wp$ function. Recall that there is no Weierstrass elliptic function when $\left(g_{2}, g_{3}\right)$ lie on the curve $\triangle=g_{2}^{3}-27 g_{3}^{2}=0$ (see Figure 4 ). By Theorems 3.2 and 4.3, the lattice $\Lambda$ is real rectangular or real rhombic. Therefore $\left(g_{2}, g_{3}\right)$-space is the space of real Weierstrass elliptic $\wp$ functions by Theorem 3.2

The discriminant divides the plane into two regions based on the shape of the lattice. If $\Delta>0$, then the lattice is rectangular and if $\Delta<0$, then the lattice is rhombic (see Figure 4). The triangular lattices lie on the vertical axis, and the square lattices lie on the horizontal axis. Thus the positive $g_{2}$ axis represents square lattices that are oriented in a rectangular position, and the negative $g_{2}$ axis represents square lattices that are oriented in a rhombic position. The invariant $g_{3}$ is positive if the lattice is vertical and negative if the lattice is horizontal. For real rhombic lattices, if $g_{2}$ is positive, then the shorter diagonal of the rhombus is shorter than its sides and we call the rhombus extreme. If $g_{2}$ is negative, then the shorter diagonal of the rhombus is longer than its sides and we call the rhombus medium (cf. [7]).

We begin with the following result that shows that the space gives a reduced holomorphic family.

Theorem 7.1. If $g_{2}^{3}-27 g_{3}^{2} \neq 0$ and $g_{2}, g_{3} \in \mathbb{R}$, then $\left(g_{2}, g_{3}\right)$-space, representing the maps $\wp_{\Lambda}$ with invariants $\left(g_{2}, g_{3}\right)$, is a reduced holomorphic family of maps.

Proof. Assume $\Lambda_{1}$ and $\Lambda_{2}$ are real lattices, with corresponding maps $\wp_{1}$ and $\wp_{2}$ having associated invariants $\left(g_{2,1}, g_{3,1}\right)$ and $\left(g_{2,2}, g_{3,2}\right)$ respectively. Assume in addition that $\wp_{1}$ is conformally conjugate to $\wp_{2}$. If $g_{2,1}=0$, then $\Lambda_{1}$ is triangular and so is $\Lambda_{2}$; applying Corollary 6.5 we have that $g_{3,1}=g_{3,2}$. Otherwise, by Lemma 6.3 


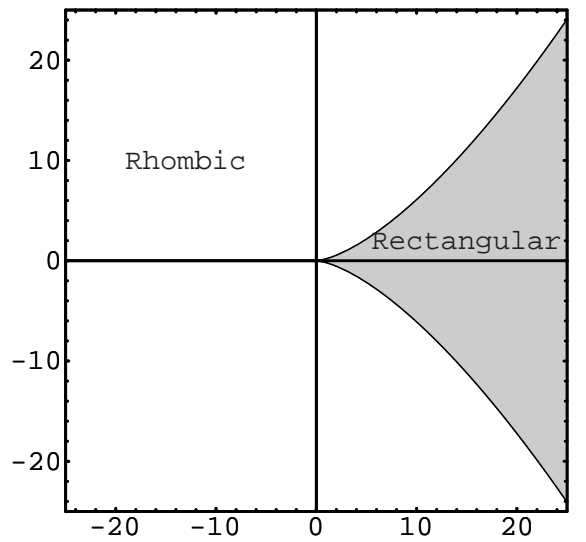

Figure 4. Shapes of lattices in real $\left(g_{2}, g_{3}\right)$-space

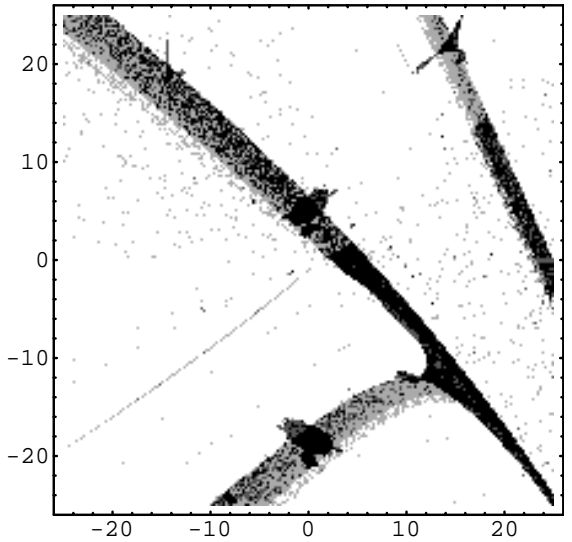

Figure 5. Real $\left(g_{2}, g_{3}\right)$-space colored according to number of attracting cycles

$\Lambda_{2}=k \Lambda_{1}$ and $\left(g_{2,2}, g_{3,2}\right)$ lie on the real algebraic curve $y^{2}=x^{3} g_{3,1}^{2} / g_{2,1}^{3}$. Equivalently, $g_{2,2}=k^{-4} g_{2,1}$ and $g_{3,2}=k^{-6} g_{3,1}$. Since all the invariants are real, however, this implies that $k^{-4}$ and $k^{-6}$ are both real. By Theorem 6.5 it follows that $k= \pm 1$ so $\Lambda_{1}=\Lambda_{2}$.

The real $\left(g_{2}, g_{3}\right)$ parameter space includes families of lattices that are similar to each other with respect to multiplication by $k \in \mathbb{R}-\{0\}$. In particular, if we fix any real pair $\left(g_{2}, g_{3}\right)$ with corresponding lattice $\Lambda$, then all parameters that lie on the planar curve $y^{2}=\left(g_{3}(\Lambda)\right)^{2} x^{3} /\left(g_{2}(\Lambda)\right)^{3}$ represent lattices similar to $\Lambda$.

Figure 5 is a coloring of parameter space based on the number of critical values that converge to attracting cycles, where white indicates no critical values converge to cycles, light grey means that one critical value does, dark grey means two critical values head to cycles, and black means three do.

Figure 6 is a closer examination of a set in Figure 5 Recall that the triangular lattices lie along the vertical axis.

Theorems 8.3 and 9.3 will tell us how to move along the triangular or square axes to find parameters corresponding to maps which have real superattracting fixed points. In fact, for any real lattice shape we can move along the curve $y^{2}=$ $\left(g_{3}(\Lambda)\right)^{2} x^{3} /\left(g_{2}(\Lambda)\right)^{3}$ until we find a parameter with a real superattracting fixed point by using the homogeneity property.

Lemma 7.2. Let $\Gamma$ be a real lattice such that $\gamma / 2$ is the smallest positive real critical point and $e_{r}$ is the largest real critical value. If $m$ is any odd integer and $k=$ $\sqrt[3]{2 e_{r} /(m \gamma)}$ (taking the real root), then the lattice $\Lambda=k \Gamma$ has a real superattracting fixed point at $m k \gamma / 2$.

Proof. Clearly, $\Lambda$ is a real lattice. The homogeneity property implies that $k \gamma / 2$ is a real critical point for $\wp_{\Lambda}$. Since $m$ is odd, periodicity implies that $\wp_{\Lambda}(m k \gamma / 2)=$ $\wp_{\Lambda}(k \gamma / 2)$. Further, the homogeneity property implies that

$$
\wp_{\Lambda}\left(\frac{k \gamma}{2}\right)=\wp_{k \Gamma}\left(\frac{k \gamma}{2}\right)=\frac{1}{k^{2}} \wp_{\Gamma}\left(\frac{\gamma}{2}\right)=\frac{e_{r}}{k^{2}}=\frac{m k \gamma}{2} .
$$




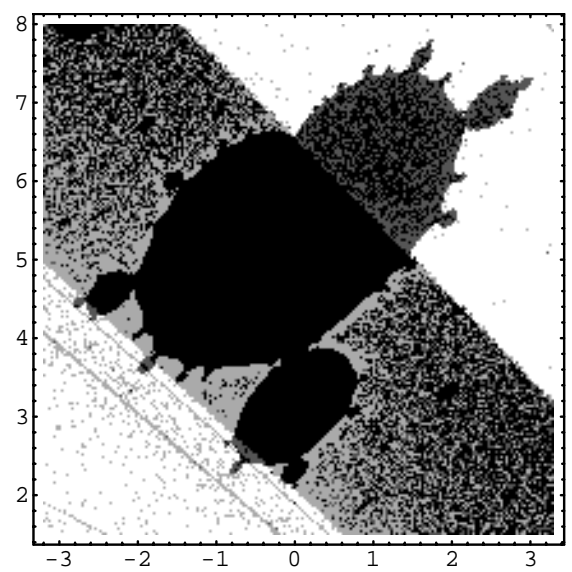

Figure 6. Blow up of real $\left(g_{2}, g_{3}\right)$-space

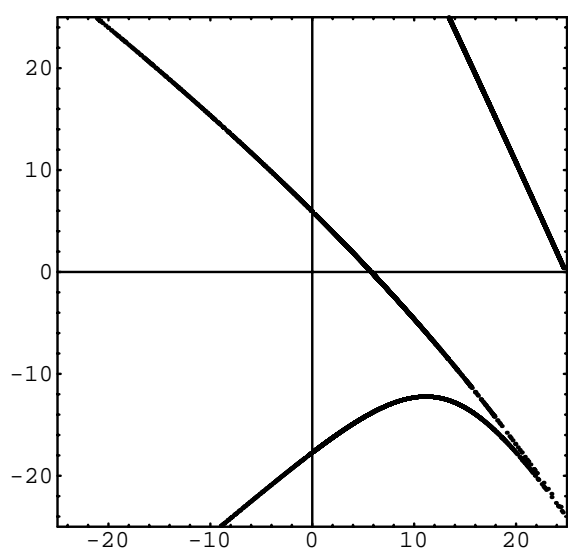

FiguRE 7. Parameters in $\left(g_{2}, g_{3}\right)$-space with a real superattracting critical point

Figure 7 shows some of the parameter values in $\left(g_{2}, g_{3}\right)$ space which have a real superattracting critical point. We can also use the homogeneity property to find lattices that have a critical value that is a lattice point and hence a pole.

Lemma 7.3. Let $\Gamma$ be a real lattice such that $\gamma / 2$ is the smallest positive real critical point and $e_{r}$ is the largest real critical value. If $m$ is any integer and $k=\sqrt[3]{e_{r} /(m \gamma)}$ (taking the real root), then the lattice $\Lambda=k \Gamma$ has $e_{1, \Lambda}=m \lambda$ and thus $e_{1, \Lambda}$ is a pole.

In [9] we constructed examples of lattices such that all critical values were prepoles and thus the Julia set is the entire sphere. These examples lie along the real and imaginary axes in $\left(g_{2}, g_{3}\right)$ space. Here we use Lemma 7.3 to find similar examples off the axes in $\left(g_{2}, g_{3}\right)$ space. 


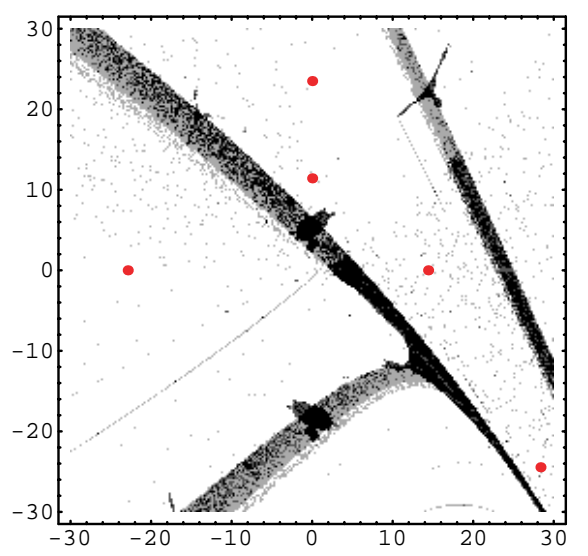

Figure 8. Parameters in $\left(g_{2}, g_{3}\right)$-space where $J\left(\wp_{\Lambda}\right)=\mathbb{C}_{\infty}$

Theorem 7.4. Let $a \neq 0,1,-2$ be an integer, and let $g_{2}(\Gamma)=4\left(a^{2}+a+1\right) / a^{2}$ and $g_{3}(\Gamma)=-4(a+1) / a^{2}$. If $m=j a$, where $j$ is any nonzero integer and $k=\sqrt[3]{1 /(m \gamma)}$ (taking the real root), then the Weierstrass elliptic function on the lattice $\Lambda=k \Gamma$ has Julia set equal to the entire sphere.

Proof. Using Equations (2) and (5) we have that

$$
e_{1, \Gamma}=1, \quad e_{2, \Gamma}=\frac{-1-a}{a}, e_{3, \Gamma}=\frac{1}{a} .
$$

If $m=j a$, then by Lemma 7.3 and Equation (3) we have that

$$
e_{1, \Lambda}=j a \lambda, \quad e_{3, \Lambda}=j \lambda .
$$

By Equation (5), $e_{2, \Lambda}$ must be a lattice point as well. Since all critical values are prepoles, the Julia set must be the entire sphere.

Figure 8 shows the locations of some of the parameters from Theorems 7.4 and 8.9 in the $\left(g_{2}, g_{3}\right)$ parameter plane. The Weierstrass elliptic functions at the parameters colored red have a Julia set equal to the entire sphere.

\section{Triangular lattices: PARAMETER SPACE AND Julia SETS}

In this section, we focus on the parameter plane for Weierstrass elliptic functions on triangular lattices. While this shape represents only one point in modular space, the results indicate the richness of the dynamics even on this small scale.

Since $g_{2}=0$ for any triangular lattice (see Proposition 4.2), we can parametrize all Weierstrass elliptic functions on triangular lattices by the complex $g_{3}$ plane minus the origin. We investigate the $g_{3}$ plane then, as it is more natural than $k$-space. We begin with the analog of Proposition 6.5 for $g_{3}$ space.

Theorem 8.1. For triangular lattices $\Gamma_{1}$ and $\Gamma_{2}, \wp_{\Gamma_{1}}$ is conformally conjugate to $\wp_{\Gamma_{2}}$ if and only if $g_{3}\left(\Gamma_{1}\right)=g_{3}\left(\Gamma_{2}\right)$ if and only if $\Gamma_{1}=\Gamma_{2}$.

Proof. Since the lattices are both the same shape, $\Gamma_{2}=k \Gamma_{1}$ for some $k \in \mathbb{C} \backslash\{0\}$. By Lemma 4.2 $g_{3}\left(\Gamma_{2}\right)=k^{-6} g_{3}\left(\Gamma_{1}\right)$. From Theorem 6.2 and Corollary 6.5 we have 


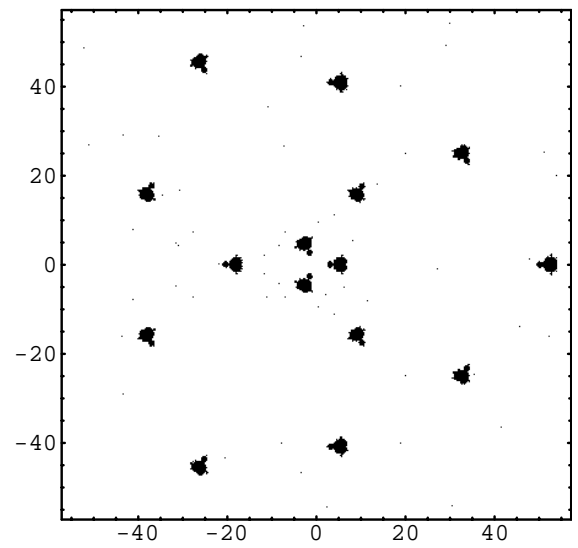

Figure 9. The $g_{3}$ parameter plane

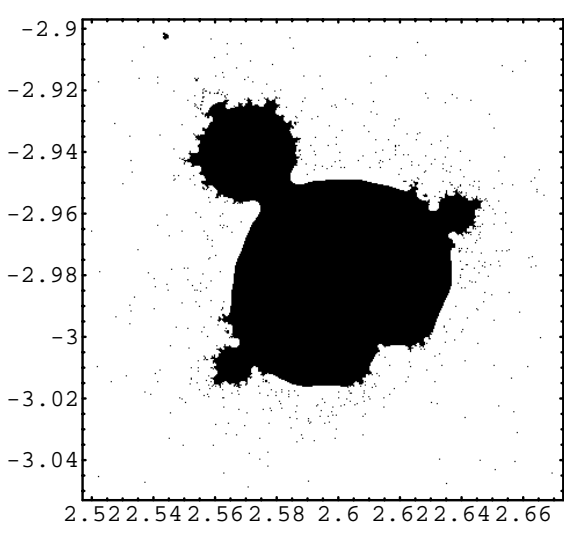

Figure 10. A period 9 Mandelbrot set

that $\wp_{\Gamma_{1}}$ is conformally conjugate to $\wp_{\Gamma_{2}}$ if and only if

$$
k=e^{j \pi i / 3}, \quad j=1, \ldots, 6,
$$

which occurs if and only if $g_{3}\left(\Gamma_{2}\right)=k^{-6} g_{3}\left(\Gamma_{1}\right)=g_{3}\left(\Gamma_{1}\right)$. Since $g_{2}=0$ for triangular lattices, $g_{3}\left(\Gamma_{2}\right)=g_{3}\left(\Gamma_{1}\right)$ if and only if $\Gamma_{2}=\Gamma_{1}$.

Corollary 8.2. The $g_{3}$-plane minus the origin gives a reduced holomorphic family of Weierstrass elliptic $\wp$ functions for triangular lattices.

Definition 8.1. We denote by $\Lambda=\left[\lambda, e^{2 \pi i / 3} \lambda\right]$, where $\lambda \in \mathbb{R}, \lambda>0$, the lattice associated with the invariants $g_{2}=0, g_{3}=4$. We call $\Lambda$ the standard triangular lattice. By Proposition 4.2(2), the critical values of $\wp_{\Lambda}$ are the cube roots of unity; let $e_{1, \Lambda}=1$.

By Proposition 5.3 for a triangular lattice, either no critical values converge to periodic cycles or all three critical values iterate toward nonrepelling cycles, so the $g_{3}$ parameter space needs only two colors to illustrate this dichotomy. Figure 9 shows the parameters for which the critical values converge to attracting cycles. There are many other smaller sets that can only be seen upon zooming in more closely. Figure 10 illustrates one of these sets. In Figure 10, parameters inside the main cardioid have attracting 9-cycles.

We begin our study with superattracting fixed points because they usually form the center of the cardioids of Mandelbrot-like sets. It is clear that any lattice $\Gamma=\left[\gamma, e^{2 \pi i / 3} \gamma\right]$ for which $\wp_{\Gamma}(\gamma / 2) \in \gamma / 2+\Gamma$ has one, hence three, superattracting fixed points.

Theorem 8.3. Let $\Lambda$ be the standard triangular lattice. For any $m, n \in \mathbb{Z}$, if

$$
k=\left(\frac{1}{(\lambda / 2)+m \lambda+n \lambda e^{2 \pi i / 3}}\right)^{1 / 3},
$$

then $\Gamma=k \Lambda$ has exactly three superattracting fixed points. These parameters are located at $g_{3}(\Gamma)=4\left(\frac{\lambda}{2}+m \lambda+n \lambda e^{2 \pi i / 3}\right)^{2}$. 

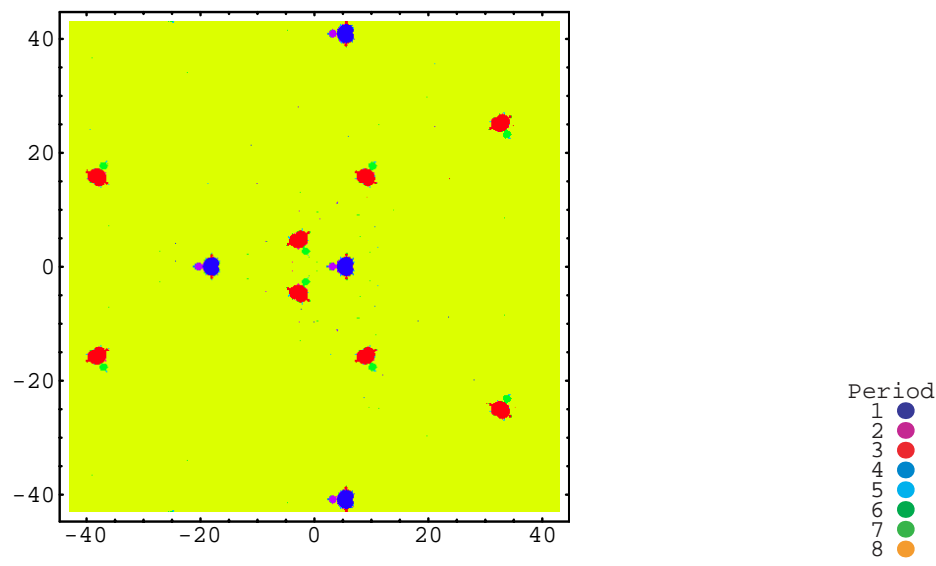

Figure 11. The $g_{3}$ parameter plane shaded according to the length of the attracting cycle

Proof. Using Equation (3) we see that $\wp_{\Gamma}(\gamma / 2)=\gamma / 2+m \gamma+n \gamma e^{2 \pi i / 3}$, so $\gamma / 2+$ $m \gamma+n \gamma e^{2 \pi i / 3}$ is a superattracting fixed point. Using Proposition [5.3, we have three superattracting fixed points.

The location of these parameters in the triangular parameter plane is given by Lemma 4.2

$$
g_{3}(\Gamma)=\frac{1}{k^{6}} g_{3}(\Lambda) .
$$

We can therefore find infinitely many points in the $g_{3}$ parameter plane where the Weierstrass elliptic function has a superattracting fixed point. These parameters are located in the centers of the red cardioids in Figure 11, Figure 11] is a coloring of Figure 9 in which attracting cycles are shaded according to the length of the cycle.

We can also prove the existence of infinitely many positive real values of $g_{3}$ for which the triangular Weierstrass elliptic function has three superattracting twocycles.

Theorem 8.4. There exist infinitely many triangular lattices $\Gamma$ such that

$$
e_{1} \rightarrow a \rightarrow e_{1}, \quad e_{2} \rightarrow a e^{2 \pi i / 3} \rightarrow e_{2}, \quad e_{3} \rightarrow a e^{4 \pi i / 3} \rightarrow e_{3},
$$

where $a \neq e_{1}$.

Proof. Let $\Lambda$ be the standard triangular lattice. Using the tables in $[21, \lambda \approx$ $2.42 \ldots$, and so $2<\lambda<3$. Recall that $\wp_{\Lambda}(\lambda / 2)=e_{1, \Lambda}=1$. By Theorem $4.3 \wp_{\Lambda}$ maps the real line to the real line.

If $\Gamma=k \Lambda=\left[k \lambda, k e^{2 \pi i / 3} \lambda\right]=\left[\gamma, e^{2 \pi i / 3} \gamma\right]$, then $\gamma / 2$ is a critical point of $\wp_{\Gamma}$. Using Equation (3), we have

$$
\wp_{\Gamma}\left(\frac{\gamma}{2}\right)=\wp_{k \Lambda}\left(\frac{k \lambda}{2}\right)=\frac{1}{k^{2}} \wp_{\Lambda}\left(\frac{\lambda}{2}\right)=\frac{1}{k^{2}} .
$$

Thus $e_{1, \Gamma}=1 / k^{2}$. 
We want to show the existence of $k>0$ such that $\wp_{\Gamma}\left(e_{1, \Gamma}\right)=\wp_{\Gamma}\left(1 / k^{2}\right)=m \gamma / 2$ where $m$ is odd and $m>3$. Using homogeneity, this is equivalent to

$$
\frac{1}{k^{2}} \wp_{\Lambda}\left(\frac{1}{k^{3}}\right)=\frac{m \gamma}{2}
$$

or $\wp_{\Lambda}\left(1 / k^{3}\right)=m k^{3} \lambda / 2$.

We define functions $g(k)=\wp_{\Lambda}\left(1 / k^{3}\right)$ and $f(k)=m k^{3} \lambda / 2$. We let $k$ range over the interval $[r, s]=[\sqrt[3]{1 / \lambda}, \sqrt[3]{2 / \lambda}]$. Since $2<\lambda<3$, we have that $r>.5$ and $s<1$. Note that $r$ is a pole of $g$, and $s$ is a critical point of $g$, and $g$ is monotone decreasing on $[r, s]$. Since $r$ is a pole of $g$, we can find a small number $\delta$ such that $g(r+\delta)>m \lambda / 2$. Clearly, $r+\delta<1$ since $s<1$. Then $f(r+\delta)<m \lambda / 2$. Thus $g-f>0$ at $k=r+\delta$.

We know that $g(s)=\wp_{\Lambda}(\lambda / 2)=1$. Since $\lambda>2$ and $s>\sqrt[3]{1 / 3}$, we have $f(s)>m(\sqrt[3]{1 / 3})^{3}>1$. Thus $g-f<0$ at $k=s$. Since both $g$ and $f$ are continuous on the interval $[r+\delta, s]$, the Intermediate Value Theorem implies that there is a $k \in(r+\delta, s)$ such that $g(k)=f(k)$. This implies the existence of a triangular lattice $\Gamma$ such that $e_{1, \Gamma}$ is periodic of period 2. By Theorem 4.3 since $e_{1, \Gamma}$ is real, $\wp_{\Gamma}\left(e_{1, \Gamma}\right)$ is real. Since $\Lambda$ is triangular, $e_{2, \Gamma}$ and $e_{3, \Gamma}$ are periodic with period two.

In Figure 11, not all of the cardioids are colored red. Theorems 8.6 and 8.7 below explain the dynamics in these cardioids. We begin with a lemma.

Lemma 8.5. If $\Gamma$ is a triangular lattice and $\Omega=e^{2 \pi i / 9} \Gamma$, then $g_{3}(\Omega)=e^{2 \pi i / 3} g_{3}(\Gamma)$.

Proof. We use

and Lemma 4.2 .

$$
\frac{1}{\left(e^{2 \pi i / 9}\right)^{6}}=e^{2 \pi i / 3}
$$

We show here that rotation of a triangular lattice by $e^{2 \pi i / 9}$ changes fixed points to cycles of period three.

Theorem 8.6. Suppose $\Gamma$ is a triangular lattice such that $\wp_{\Gamma}$ has a fixed point at $p$, and let $k_{1}=e^{2 \pi i / 9}$ and $k_{2}=e^{4 \pi i / 9}$. Then,

(1) if $\Omega_{1}=k_{1} \Gamma$ and $q=k_{1} p$, then $\wp_{\Omega_{1}}$ has a three cycle $q \rightarrow e^{4 \pi i / 3} q \rightarrow$ $e^{2 \pi i / 3} q \rightarrow q \ldots$

(2) if $\Omega_{2}=k_{2} \Gamma$ and $q=k_{2} p$, then $\wp_{\Omega_{2}}$ has a three cycle $q \rightarrow e^{2 \pi i / 3} q \rightarrow$ $e^{4 \pi i / 3} q \rightarrow q \ldots$

The classification of the resulting 3-cycle for $\Omega_{1}$ or $\Omega_{2}$ is the same as the classification of the fixed point for $\Gamma$.

Proof. Using homogeneity and Proposition 6.1 we have

$$
\wp_{\Omega_{1}}(q)=\wp_{k_{1} \Gamma}\left(k_{1} p\right)=\frac{1}{e^{4 \pi i / 9}} \wp_{\Gamma}(p)=e^{14 \pi i / 9} p=e^{4 \pi i / 3} q .
$$

Similarly, $\wp_{\Omega_{1}}\left(e^{4 \pi i / 3} q\right)=e^{2 \pi i / 3} q$, and $\wp_{\Omega_{1}}\left(e^{2 \pi i / 3} q\right)=q$.

Since $\Gamma$ is triangular, we know that $\left|\wp_{\Gamma}^{\prime}(p)\right|=\left|\wp_{\Gamma}^{\prime}\left(e^{2 \pi i / 3} p\right)\right|=\left|\wp_{\Gamma}^{\prime}\left(e^{4 \pi i / 3} p\right)\right|$. Using the homogeneity property for the derivative, we have that $\left|\wp_{\Omega_{1}}^{\prime}(q)\right| \cdot\left|\wp_{\Omega_{1}}^{\prime}\left(e^{2 \pi i / 3} q\right)\right|$. $\left|\wp_{\Omega_{1}}^{\prime}\left(e^{2 \pi i / 3} q\right)\right|=\left|\wp_{\Gamma}^{\prime}(p)\right| \cdot\left|\wp_{\Gamma}^{\prime}\left(e^{2 \pi i / 3} p\right)\right| \cdot\left|\wp_{\Gamma}^{\prime}\left(e^{4 \pi i / 3} p\right)\right|$. Thus the classification of the cycle is the same as the classification of the fixed point.

The proof for $\Omega_{2}$ follows similarly. 
The splitting of the three fixed points into a period 3 cycle that we saw in Theorem 8.6 holds for cycles of longer length as long as the length of the cycle is not divisible by three. If the cycle has length $n$ where $n$ is divisible by three, then it may collapse into three distinct cycles of length $n / 3$, or it might remain as an $n$-cycle, possibly containing different points in the cycle.

Theorem 8.7. Suppose $\Gamma$ is a lattice such that $\wp_{\Gamma}$ has a periodic cycle $p=$ $\left\{p_{1}, p_{2}, \ldots, p_{n}\right\}$. Let $k_{1}=e^{2 \pi i / 9}$ and $k_{2}=e^{4 \pi i / 9}$, and $\Omega_{1}=k_{1} \Gamma$ and $\Omega_{2}=k_{2} \Gamma$. Then, we have the following:

(1) If $n$ is not divisible by three, then $\wp_{\Omega_{1}}$ and $\wp_{\Omega_{2}}$ have $3 n$ cycles at $k_{1} p_{1}$ and $k_{2} p_{1}$.

(2) If $n=3 m$, then either:

(a) if $\operatorname{Arg}\left(p_{i+1} / p_{i}\right)= \pm 2 \pi / 3$ for all $i$, then $\wp_{\Omega_{i}}$ has an $n$-cycle at $k_{i} p_{1}$ and $\wp \Omega_{j}$ has an $m$ cycle at $k_{j} p_{1}$ where $i \neq j$;

(b) otherwise both $\wp_{\Omega_{1}}$ and $\wp_{\Omega_{2}}$ have $n$ cycles at $k_{1} p_{1}$ and $k_{2} p_{1}$ respectively.

The classification of the resulting cycle in $\Omega_{1}$ or $\Omega_{2}$ is the same as the classification of the cycle in $\Gamma$.

Corollary 8.8. If $\Gamma$ is a triangular lattice and $\Omega=e^{2 \pi i / 9} \Gamma$, then $J\left(\wp_{\Omega}\right)=$ $e^{2 \pi i / 9} J\left(\wp_{\Gamma}\right)$.

Proof. First, note that $\Gamma=e^{4 \pi i / 9} \Omega$. Then Theorem 8.7 implies that $z$ is a repelling periodic point of $\wp_{\Gamma}$ if and only if $e^{2 \pi i / 9} z$ is a repelling periodic point of $\wp_{\Omega}$. Since the Julia set is the closure of the repelling periodic points (see [4]), $J(\wp \Omega)=e^{2 \pi i / 9} J\left(\wp_{\Gamma}\right)$.

Lemma 8.5. Theorem 8.7 and Corollary 8.8 explain why we see triples of Mandelbrot sets lying on circles in the $g_{3}$ parameter space. Although we see $2 \pi / 3$ rotational symmetry in $g_{3}$ space, recall that we do not have conformal conjugacy by Theorem 8.1 We always find three Mandelbrot sets that lie on a circle, where one of the main cardioids signifies that the resulting Weierstrass elliptic function has an attracting fixed point, and the other two main cardioids represent elliptic functions that have attracting 3 cycles.

In Figure 12 we zoom in on a region in Figure 11. Notice that the period 3 bulbs in one Mandelbrot set can rotate to period 3 bulbs in the other two Mandelbrot sets. For example, both the parameters $g_{3}=(5.67+2.08 i)$ and $g_{3}=(5.67+2.08 i) e^{2 \pi i / 3}$ have attracting cycles of period three. (The Julia sets for these parameters are shown below.)

We use Mathematica to draw some Julia sets corresponding to specific points in the $g_{3}$ plane. For each point in the window, the algorithm colors a point yellow, blue, or green (or white, or a shade of grey) if it iterates to the (known) attracting cycle and red (or black) if it does not. Thus the red (or black) region in the following pictures represents the Julia set.

Figure 13 is an illustration of the Julia set of the Weierstrass elliptic function on the triangular lattice $\Gamma$ where $g_{3}(\Gamma) \approx 5.898$ has exactly three superattracting fixed points as shown in Theorem 8.3. The fixed points are located close to 1.138, $1.138 e^{2 \pi i / 3}$, and $1.138 e^{4 \pi i / 3}$. Points in the Fatou set are colored depending on which fixed point they are attracted to; for example, all points colored yellow (or light grey) in Figure 13 iterate to the fixed point 1.138. 

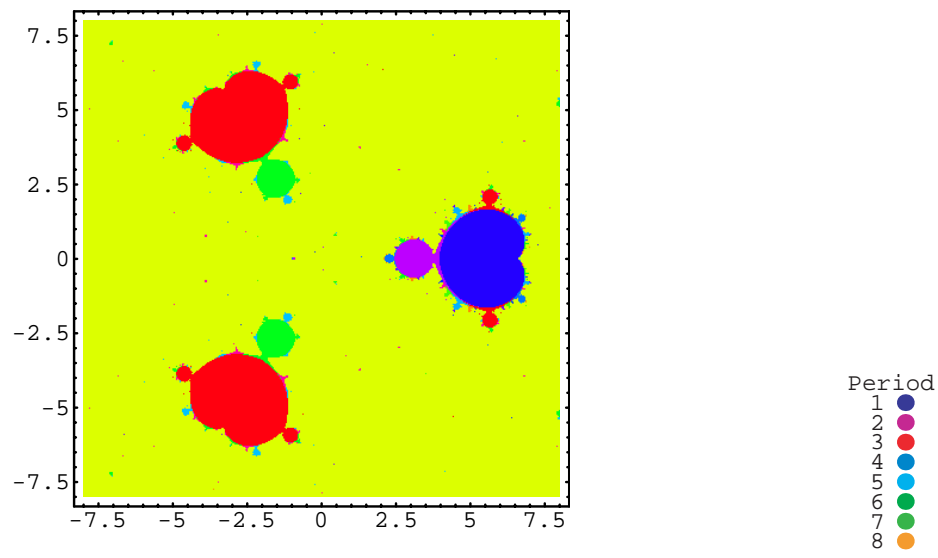

FIGURE 12. $g_{3}$-space

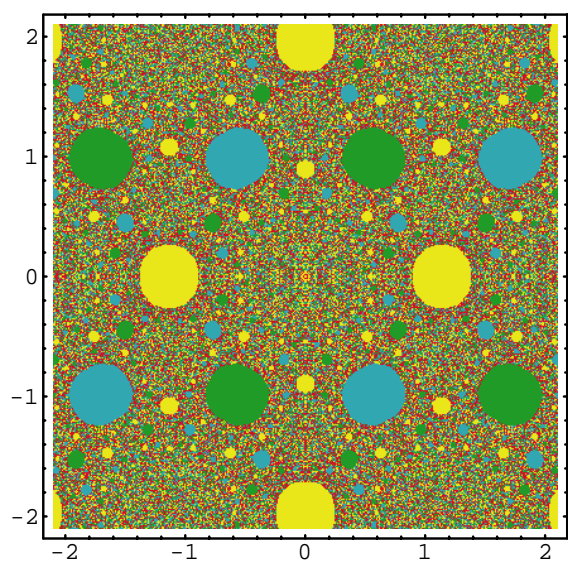

FIGURE 13. $J\left(\wp_{\Gamma}\right)$ when $g_{3} \approx 5.898$

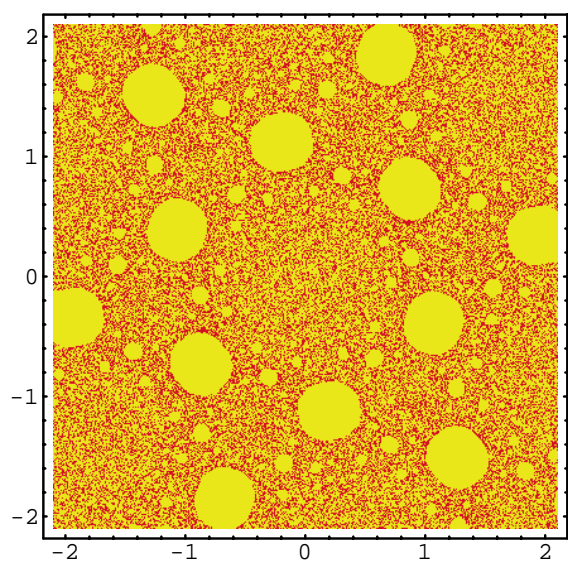

FigURE $14 . \quad J\left(\wp_{\Omega}\right)$ when $g_{3} \approx 5.898 e^{2 \pi i / 3}$

Figure 14 illustrates the Julia set of $\wp \Omega$ where $\Omega=e^{2 \pi i / 9} \Gamma$, which by Theorem 8.6 has one superattracting three cycle, and all points in the Fatou set are attracted to this three cycle. By Lemma $8.5 g_{3}(\Omega) \approx 5.898 e^{2 \pi i / 3}$. Figure 15 shows the Julia set for the parameter $g_{3} \approx 5.67+2.08 i$. In this case, there are three attracting cycles of length three in the Fatou set. One of these cycles occurs at approximately $p=\{1.139+.134 i, .989+.11 i, 1.131-.068 i\}$; all points colored blue (medium grey) in Figure 15 iterate to this cycle. There is another three cycle located at $e^{2 \pi i / 3} p$ and another at $e^{2 \pi i / 3} p$. Figure 17 shows a zoom in on the cycle $p$, where the points in the cycle are marked in navy blue (or black). Figure 16 shows the Julia set for the parameter $g_{3}=(5.67+2.08 i) e^{2 \pi i / 3}$. By Theorem $8.7(2 \mathrm{~b})$ the Fatou set in Figure [16 also has three attracting cycles of length three, and points in the Fatou set are colored depending on which cycle they are attracted to. 


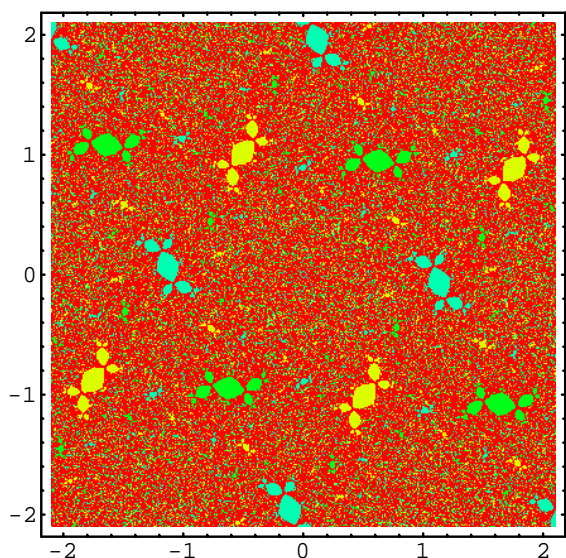

FIGURE $15 . \quad J\left(\wp_{\Gamma}\right)$ when $g_{3} \approx$ $5.67+2.08 i$

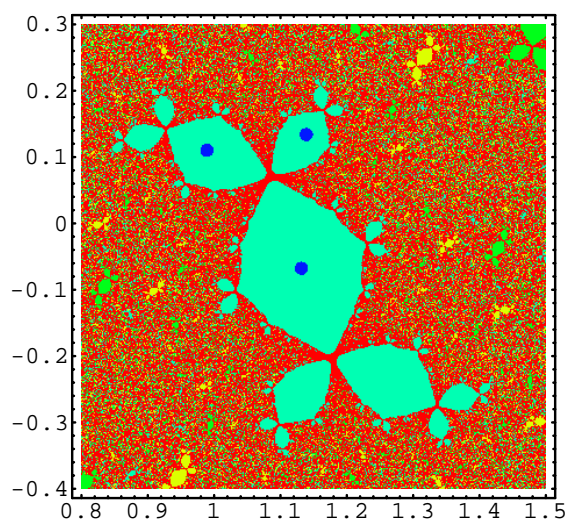

FIGURE 17. Weierstrass rabbit

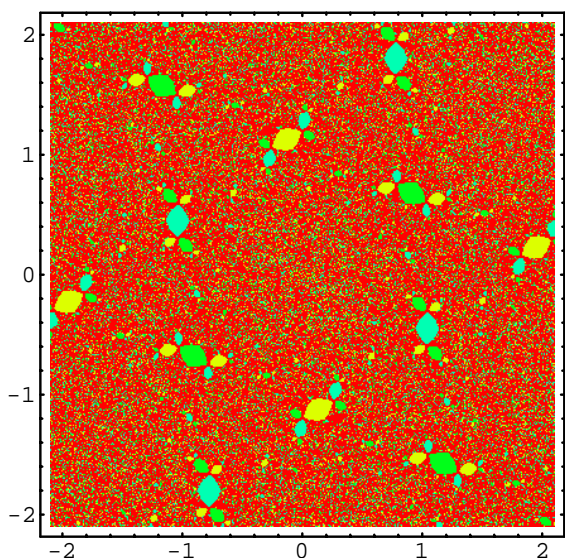

FIGURE 16. $J\left(\wp_{\Omega}\right)$ when $g_{3} \approx$ $(5.67+2.08 i) e^{2 \pi i / 3}$

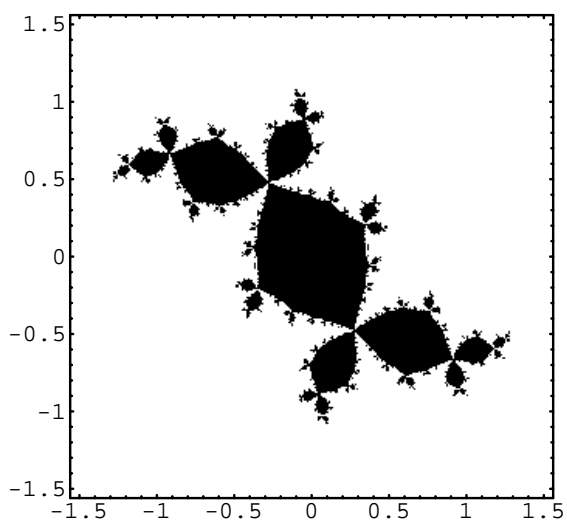

FiguRE 18. Douady rabbit

The following theorem locates $g_{3}$ parameters for which the critical values are lattice points. In this case, all critical values lie in the Julia set and have a finite forward orbit. The Fatou set is therefore empty.

Theorem 8.9. Let $\Lambda$ denote the standard triangular lattice. For any $m, n \in \mathbb{Z}$ where $m$ and $n$ are not simultaneously zero, if

$$
k=\left(\frac{1}{m \lambda+n \lambda e^{2 \pi i / 3}}\right)^{1 / 3}
$$

then $\Gamma=k \Lambda$ has Julia set equal to the entire sphere. These parameters are located at $g_{3}(\Gamma)=4\left(m \lambda+n \lambda e^{2 \pi i / 3}\right)^{2}$.

Proof. Using Equation (3) we see that $\wp_{\Gamma}(\gamma / 2)=m \gamma+n \gamma e^{2 \pi i / 3}$. Using Proposition [5.3, we see that all of the critical values are lattice points and lie in the Julia set. Therefore the Fatou set must be empty. The location of these parameters in the triangular parameter plane is given by Lemma 4.2 


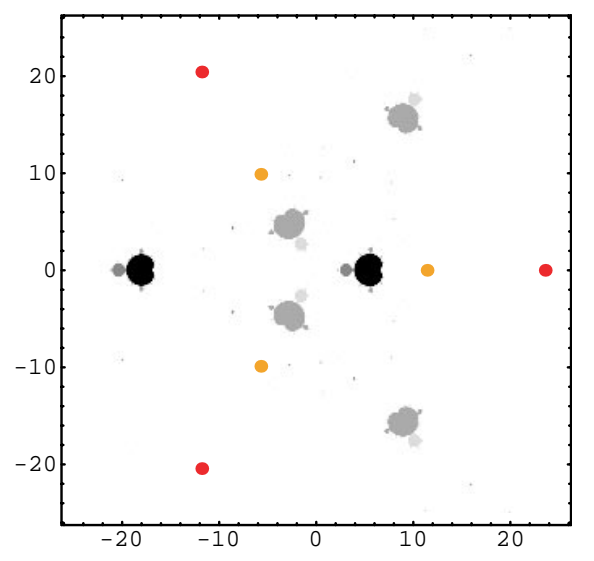

FiguRE 19. Parameters in $g_{3}$-space with $J(\wp)=\mathbb{C}_{\infty}$

In Figure 19 the parameter values given in Theorem 8.9 are colored red. We note that there are other locations in the $g_{3}$ plane where the Julia set is the entire sphere. For example, any parameter value for which the critical points eventually land on lattice points will give rise to elliptic functions with empty Fatou set. Similarly, any parameter value for which the critical points are preperiodic will have Julia set equal to the entire sphere. In [9] we proved the existence of parameter values such that $\wp_{\Lambda}$ has critical points which are preperiodic but not prepoles. These points are colored yellow in Figure 19 and were proven in 9 to correspond to Lebesgue ergodic and exact maps of the sphere.

\section{Parametrization of square lattices: $g_{2}$-SPace}

We turn to a discussion of the parameter plane for Weierstrass elliptic functions on square lattices. By Proposition 4.2, $g_{3}=0$ for any square lattice, so we can parametrize all Weierstrass elliptic functions on square lattices by the complex $g_{2}$ plane minus the origin. Although each $g_{2}$ corresponds to a unique lattice, the conjugacy given in Proposition 6.2 leads to $e^{2 \pi i / 3}$ rotational symmetry in $g_{2}$-space coming from conformal conjugacy of the mappings. Therefore, $g_{2}$-space is not a reduced space.

Theorem 9.1. For square lattices $\Gamma_{1}$ and $\Gamma_{2}, \wp_{\Gamma_{1}}$ is conformally conjugate to $\wp_{\Gamma_{2}}$ if and only if $e^{-2 \pi i / 3} g_{2}\left(\Gamma_{1}\right)=g_{2}\left(\Gamma_{2}\right)$ or $e^{2 \pi i / 3} g_{2}\left(\Gamma_{1}\right)=g_{2}\left(\Gamma_{2}\right)$.

Proof. $(\Leftarrow)$ : By Theorem 6.2 and Proposition 6.2, we have that if $\Gamma_{2}=e^{\pi i / 6} \Gamma_{1}$, then $\wp_{\Gamma_{1}}$ is conformally conjugate to $\wp_{\Gamma_{2}}$. However, by Lemma 4.2, $\Gamma_{2}=e^{\pi i / 6} \Gamma_{1}$ if and only if $e^{-2 \pi i / 3} g_{2}\left(\Gamma_{1}\right)=g_{2}\left(\Gamma_{2}\right)$. Reversing the roles of $\Gamma_{1}$ and $\Gamma_{2}$ gives the other possibility.

$(\Rightarrow)$ : Suppose that $\wp_{\Gamma_{1}}$ is conformally conjugate to $\wp_{\Gamma_{2}}$, then the conjugating map is of the form $M(z)=a z$ by Lemma 6.3. We then write $\Gamma_{2}=a \Gamma_{1}$, and it follows by Lemma 4.2 that $g_{2}\left(\Gamma_{2}\right)=a^{-4} g_{2}\left(\Gamma_{1}\right)$. The postcritical values for a square lattice always satisfy $e_{1}=-e_{2}=\sqrt{g_{2}} / 4$ and $e_{3}=0$, by Proposition 4.2. Then

$$
M\left(e_{1,1}\right)=a \sqrt{g_{2}\left(\Gamma_{1}\right)} / 4= \pm \sqrt{a^{-4} g_{2}\left(\Gamma_{1}\right)} / 4,
$$




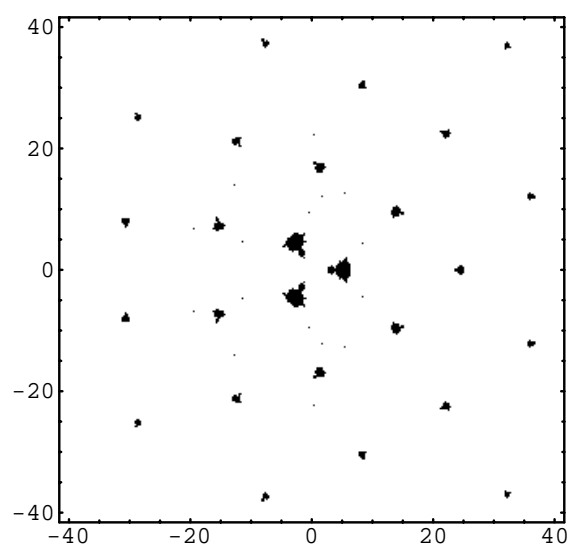

Figure 20. $g_{2}$-space

so we have

$$
\pm a^{3}=1
$$

which gives the result.

Corollary 9.2. For square lattices, the sector of $g_{2}$-space such that

$$
-\frac{\pi}{3}<\operatorname{Arg}\left(g_{2}\right) \leq \frac{\pi}{3}
$$

is a reduced holomorphic family of meromorphic maps.

Figure 20 is an illustration of the parameters for which the critical points head to attracting cycles. While Figure 20 looks much like Figure9, the structure of the parameter space of square lattices differs significantly from the triangular case due to Theorem 9.1

Changing notation from the previous section, in this section, we denote by $\Lambda=$ $[\lambda, i \lambda]$, where $\lambda \in \mathbb{R}, \lambda>0$ the lattice associated with the invariants $g_{2}=4, g_{3}=0$. By Equation (4) the critical values of $\wp_{\Lambda}$ are $0,1,-1$; let $e_{1, \Lambda}=1$. We use this lattice to orient ourselves in parameter space for square lattices, and refer to it as the standard square lattice.

It is straightforward to locate parameters which have a superattracting fixed point.

Theorem 9.3. Starting with the standard square lattice $\Lambda$, if

$$
k=\left(\frac{1}{(\lambda / 2)+m \lambda+n \lambda i}\right)^{1 / 3}
$$

(taking any of the complex roots), then $\Gamma=k \Lambda$ has a superattracting fixed point. These parameters are located at $g_{2}(\Gamma)=4\left(\frac{\lambda}{2}+m \lambda+n i \lambda\right)^{4 / 3}$.

Proof. Using the homogeneity property, we see that $\wp_{\Gamma}(\gamma / 2)=\gamma / 2+m \gamma+n \gamma i$. Using Proposition 5.4 we only have one periodic component of the Fatou set. The location of these parameters in the $g_{2}$ parameter plane is given by Lemma 4.2

Remark. Theorem 6.2 explains why any of the three cube roots may be used in Theorem 9.3 . 


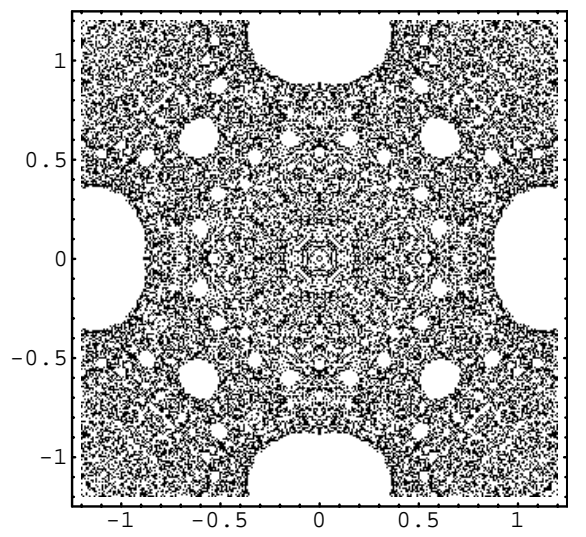

Figure 21. $J\left(\wp_{\Gamma}\right)$ when $g_{2} \approx 5.746$

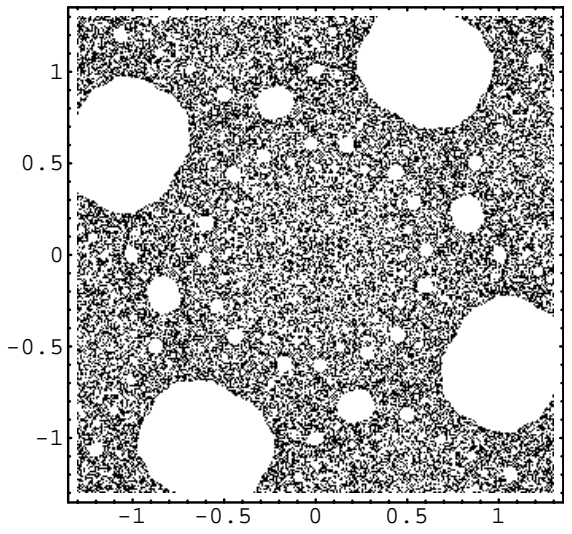

FigURE $22 . \quad J\left(\wp_{\Omega}\right)$ when $g_{2} \approx 5.746 e^{2 \pi i / 3}$

We can also prove the existence of infinitely many positive real values of $g_{2}$ for which the corresponding map has exactly one superattracting two-cycle.

Theorem 9.4. There exists infinitely many square lattices $\Gamma$ such that $e_{1} \rightarrow a \rightarrow$ $e_{1}$, where $a \neq e_{1}$.

Proof. Let $\Lambda=[\lambda, i \lambda]$ be the standard square lattice. Using the tables in [21], $\lambda \approx 2.62$, so $2<\lambda<3$. Recall that $\wp_{\Lambda}(\lambda)=e_{1, \Lambda}=1$. The proof of the existence of lattices with superattracting two-cycles follows from the Intermediate Value Theorem argument given in the proof of Theorem 8.4. By Proposition 5.4 there is only one possible Fatou cycle for square lattices.

We have shown that the triples of Mandelbrot sets lying on circles in $g_{2}$-space represent conformally conjugate Weierstrass elliptic functions defined on square lattices that are rotations by $e^{4 \pi i / 3}$. Figure 21 shows the Julia set of the Weierstrass elliptic function on a square lattice $\Gamma$ with $g_{2} \approx 5.746$ that has a real superattracting fixed point at 1.198 given by Theorem 9.3 . Figure 22 illustrates the resulted rotated Julia set on the lattice $\Omega=e^{4 \pi i / 3} \Gamma$ described by Theorem 6.2, so that $g_{2} \approx 5.746 e^{2 \pi i / 3}$. The function $\wp_{\Omega}$ has a superattracting fixed point at $1.198 e^{2 \pi i / 3}$.

We can also locate parameters where the Julia set is the entire sphere.

Theorem 9.5. Let $\Lambda$ denote the standard square lattice. For any $m, n \in \mathbb{Z}$ where $m$ and $n$ are not simultaneously zero, if

$$
k=\left(\frac{1}{m \lambda+n \lambda i}\right)^{1 / 3}
$$

then $\Gamma=k \Lambda$ has Julia set equal to the entire sphere. These parameters are located at $g_{2}(\Gamma)=4(m \lambda+n \lambda i)^{4 / 3}$.

Proof. Using Equation (3) we see that $\wp_{\Gamma}(\gamma / 2)=m \gamma+n \gamma i$. Using Proposition 5.4 we see that all of the critical values are lattice points and lie in the Julia set, and thus the Fatou set must be empty. The location of these parameters in the square parameter plane is given by Lemma 4.2,

In Figure 23, the parameter values given in Theorem 9.5 are colored red. 


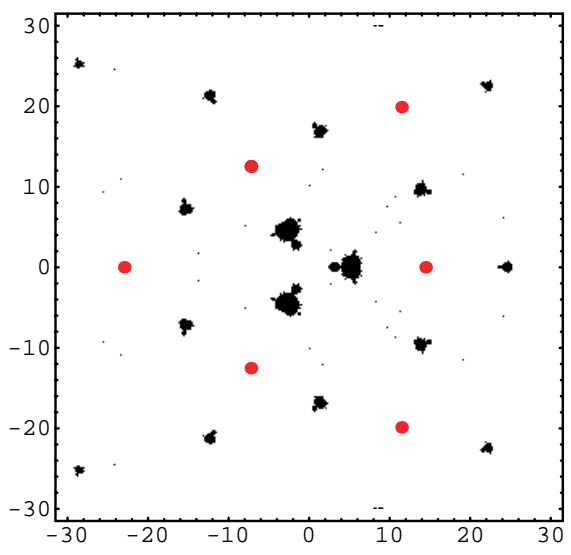

FiguRE 23. Parameters in $g_{2}$-space with $J(\wp)=\mathbb{C}_{\infty}$

\section{TORAL BANDS OCCUR FOR A RECTANGULAR LATtiCE}

In this section we show the existence of a map with a Fatou component containing a toral band. It is not known if there are lattices $\Lambda$ for which $J\left(\wp_{\Lambda}\right)$ is a Cantor set, but the existence of a toral band is necessary for one to exist. We consider first an arbitrary real rectangular lattice $\Lambda=\left[\lambda_{1}, \lambda_{2} i\right]$; assume that the lattice is not square, so $|\tau|>1$.

By [7] we know that $\wp_{\Lambda}$ is real on the boundary of the small rectangle made by the half periods of $\Lambda$. We also have that $e_{1}$ is positive real, $e_{2}$ is negative real, and $e_{3}$ is positive real if $\Lambda$ is a horizontal rectangle (i.e., $\lambda_{1}>\lambda_{2}$ ) and negative if $\Lambda$ is vertical $\left(\lambda_{1}<\lambda_{2}\right)$, by Equation (5).

In particular, for horizontal real rectangular lattices,

$$
e_{2}<0<e_{3}<e_{1},
$$

and $g_{3}<0$. As we move counterclockwise from the origin around the half-period rectangle, the map $\wp_{\Lambda}$ decreases from $+\infty$ to $e_{1}$ at $\lambda_{1} / 2$, then decreases from $e_{1}$ to $e_{3}$ along the vertical segment from $\lambda_{1} / 2$ to $\lambda_{1} / 2+i \lambda_{2} / 2$; heading horizontally to the imaginary axis brings us from $e_{3}$ to $e_{2}<0$. Finally, going down the imaginary axis back to the origin, $\wp_{\Lambda}$ decreases from $e_{2}$ to $-\infty$. In fact, the map $\wp_{\Lambda}$ maps the half-period rectangle injectively onto the closed lower (or upper) half plane except at the point 0 .

We therefore have the following result.

Theorem 10.1. If $\Lambda$ is a real rectangular lattice which is horizontal, and if there exists an attracting or parabolic periodic orbit such that $e_{3}$ and $e_{1}$ lie in the same component of the attracting basin of the cycle, then $F\left(\wp_{\Lambda}\right)$ contains a toral band.

Proof. Under the assumption that $\Lambda=\left[\lambda_{1}, i \lambda_{2}\right]$ is real rectangular and horizontal, we have that $g_{3}<0$, so $e_{3}>0$. This means that the preimage of 0 on the boundary of the half-lattice rectangle lies on the horizontal segment between $i \lambda_{2} / 2$ and $\lambda_{1} / 2+i \lambda_{2} / 2$.

By [9] (Proposition 6.10), all nonrepelling orbits are real and lie in $\left[e_{1}, \infty\right)$. Our hypothesis implies that the entire interval $I=\left[e_{3}, e_{1}\right]$ is contained in the same Fatou 
component; because $F\left(\wp_{\Lambda}\right)$ is open, and by compactness of $I$, an open strip $V \subset \mathbb{C}$ containing $I$ is also in the Fatou set. Therefore the preimage of $V$ overlapping the half rectangle is in the Fatou set as well. Since $\wp_{\Lambda}^{-1}(I) \supset\left[\lambda_{1} / 2, \lambda_{1} / 2+i \lambda_{2} / 2\right]$ (vertical line segment), and is open, we have an open vertical strip in the Fatou set centered along the half-lattice vertical lines. By symmetry of the Fatou set, this open strip extends vertically throughout the entire fundamental parallelogram for $\Lambda$ and then through all lattices. Therefore, it projects down under $\pi$ to a toral band.

Theorem 10.2. Toral band Fatou components exist.

Proof. We give an example satisfying the conditions of Theorem 10.1 We begin with the rectangular lattice $\Lambda=\left[\lambda_{1}, \lambda_{2}\right]$ with $\lambda_{1}>0$ associated with the invariants $g_{2}(\Lambda)=11, g_{3}(\Lambda)=-7$. Solving Equation (4), we obtain $e_{1, \Lambda}=1, e_{2, \Lambda}=$ $(-1-2 \sqrt{2}) / 2$, and $e_{3, \Lambda}=(-1+2 \sqrt{2}) / 2$. If $k=\sqrt[3]{2 / \lambda_{1}}$, then $\Gamma=\left[\gamma_{1}, \gamma_{2}\right]=$ $\left[k \lambda_{1}, k \lambda_{2}\right]$ has a superattracting fixed point at $e_{1, \Gamma}=\gamma_{1} / 2=\left(\lambda_{1} / 2\right)^{2 / 3}$ by Lemma 7.2. By Lemma 4.2, $g_{2}(\Gamma)=g_{2}(\Lambda) / k^{4}=11\left(\lambda_{1} / 2\right)^{4 / 3}$.

Using Equation (6), we have

$$
d_{1, \Gamma}^{2}=3 e_{1, \Gamma}^{2}-\frac{g_{2}(\Gamma)}{4}=3\left(\left(\frac{\lambda_{1}}{2}\right)^{2 / 3}\right)^{2}-\frac{11\left(\frac{\lambda_{1}}{2}\right)^{4 / 3}}{4}=\frac{\left(\frac{\lambda_{1}}{2}\right)^{4 / 3}}{4},
$$

and thus

$$
d_{1, \Gamma}=\frac{\left(\frac{\lambda_{1}}{2}\right)^{2 / 3}}{2}=\frac{\gamma_{1}}{4} .
$$

By Equation (7) we know that $\wp_{\Gamma}\left(\gamma_{1} / 4\right)=e_{1, \Gamma}+d_{1, \Gamma}=3 \gamma_{1} / 4$. By periodicity, with respect to $\Gamma$, this forces $\wp_{\Gamma}\left(3 \gamma_{1} / 4\right)=3 \gamma_{1} / 4$. Thus we have a superattracting fixed point at $\gamma_{1} / 2$ and another fixed point at $3 \gamma_{1} / 4$.

Since $\wp_{\Gamma}$ is strictly increasing on $\left[\gamma_{1} / 2, \gamma\right]$, the fixed point at $3 \gamma_{1} / 4$ must be repelling. However, $\wp_{\Gamma}$ is symmetric with respect to $\gamma_{1} / 2$, and thus all points in the interval $\left(\gamma_{1} / 4,3 \gamma_{1} / 4\right)$ must be attracted to the superattracting fixed point at $\gamma_{1} / 2$. By Proposition 4.1 $\gamma_{1} / 4<e_{3, \Gamma}<3 \gamma_{1} / 4$, and so $e_{1, \Gamma}$ and $e_{3, \Gamma}$ lie in the same Fatou component. By Theorem 10.1 $\wp_{\Gamma}$ has a toral band.

Figure 24] is a picture of the Julia set of the elliptic function exhibited in Theorem 10.2. Here, the lattice $\Lambda=[3.0184,1.5092 i]$ is horizontal rectangular. There is a superattracting fixed point at $e_{1}=1.5092$, and $e_{3}=1.3797$ lies in the superattracting component. We note that this example occurs at the parameter $k=1.5092 i$ in the $k$-space shown in Figure 2 The coloring in the algorithm leaves dark blue the points in the Julia set and puts a red neighborhood around the superattracting fixed point. The other points are colored according to how many iterations it takes to enter the red neighborhood.

\section{EXAMPLES FROM OTHER ELLIPTIC FUNCTIONS}

In a recent preprint [10, the authors construct an elliptic function of the form $f_{\Gamma}(z)=\left(\wp_{\Gamma}(z)+1\right) / \wp_{\Gamma}(z)$ with a real square lattice $\Gamma=[\gamma, \gamma i] ; \Gamma$ is the period lattice for both $\wp_{\Gamma}$ and $f_{\Gamma}$. We show that for a specific choice of $\Gamma$ (as well as for nearby choices), this function has a Julia set which is a Cantor set.

Since an explicit formula for a fixed point of $\wp_{\Lambda}$ is difficult to write down in terms of the $\Lambda$ or the invariants $g_{2}(\Lambda)$ and $g_{3}(\Lambda)$, proving the existence of a Siegel disk is tricky. However, if one considers elliptic functions of the form $\wp_{\Lambda}+b$, for $b \in \mathbb{C}$, 


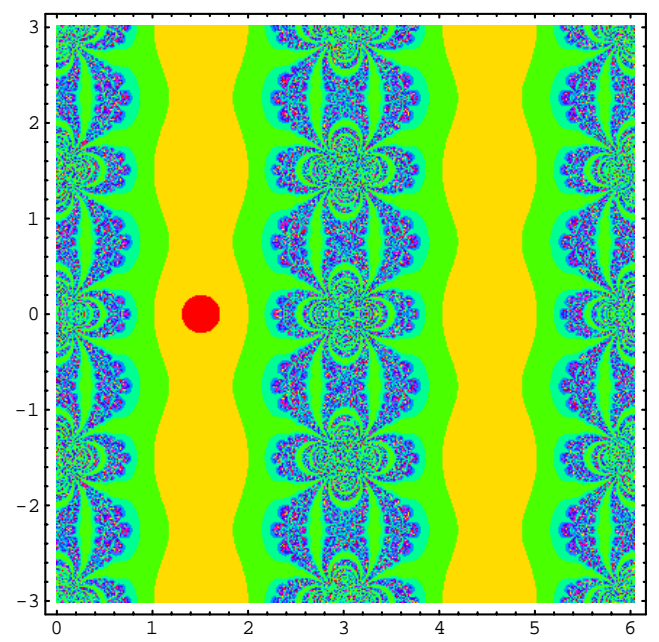

FiguRE 24. Fatou band from Theorem 10.2

then one can construct fixed points with arbitrary derivative. From the following result, we can conclude the existence of Siegel disks and Cremer points for elliptic maps.

Proposition 11.1. For any lattice $\Lambda$, and any $\alpha \in \mathbb{C}$, there exists a constant $b \in \mathbb{C}$ such that the map $f_{\Lambda}=\wp_{\Lambda}+b$ has a fixed point with derivative $\alpha$.

Proof. For any choice of lattice $\Lambda$, the map $\wp_{\Lambda}^{\prime}$ maps surjectively onto $\mathbb{C}$. Therefore, given $\alpha \in \mathbb{C}$, we choose and fix a point $z_{o}$ such that $\wp_{\Lambda}^{\prime}\left(z_{o}\right)=\alpha$. Setting $b_{o}=$ $\wp_{\Lambda}\left(z_{o}\right)$, we choose $b=-b_{o}+z_{o}$. The function $f_{\Lambda}=\wp_{\Lambda}+b$ clearly has the desired property at $z_{o}$.

Example. If we start with the standard triangular lattice $\Lambda$, then $g_{2}=0$ and $g_{3}=4$. We fix $\alpha=e^{2 \pi i(1 / 4)^{(1 / 3)}}$; now using the fact that

$$
\left(\wp_{\Lambda}^{\prime}\right)^{2}=4\left(\wp_{\Lambda}^{3}-1\right)
$$

we solve the equation:

$$
\left(\frac{\alpha^{2}}{4}+1\right)^{1 / 3}=\wp_{\Lambda}(z)
$$

for $z=z_{0}$, and then $b_{0}=\wp_{\Lambda}\left(z_{0}\right)$. The resulting map $f=\wp_{\Lambda}+b$ with $b \approx$ $-2.1151+0.0084462 i$ has a neutral fixed point and Siegel disk centered at $z_{0} \approx$ $-1.07084+0.0747154 i$. In Figure 25] we show a computer rendition of the Siegel disk centered at $z_{0}$. 


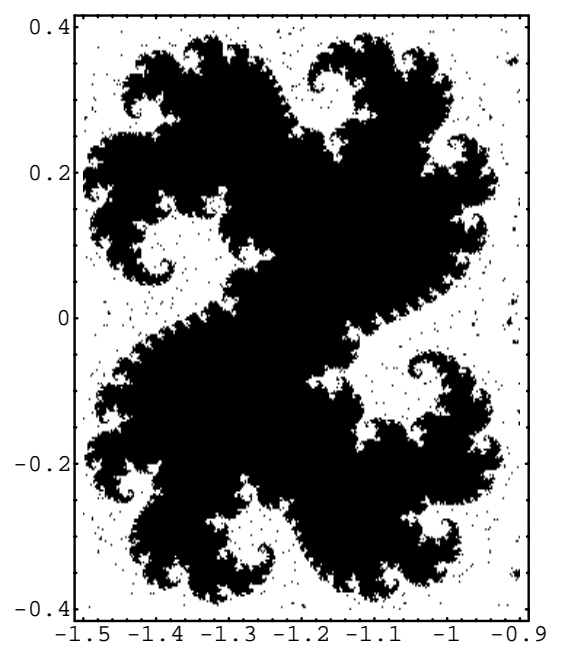

FIGURE 25. Siegel disk for $f(z)=\wp_{\Lambda}(z)+b$ for a triangular lattice $\Lambda$

\section{REFERENCES}

[1] Baker, I. N., Kotus, J., Lü, Y. (1991), Iterates of meromorphic functions, I, ETDS 11: 241248. MR 92m:58113

[2] — (1990), Iterates of meromorphic functions, III, ETDS 11: 603-618. MR 92m:58115

[3] , (1992), Iterates of meromorphic functions IV: Critically finite functions, Results Math. 22: 651-656. MR 94c:58166

[4] Bergweiler, W. (1993), Iteration of meromorphic functions, Bull. Amer. Math. Soc. 29, no. 2: 151-188. MR 94c:30033

[5] Devaney, R., Keen, L. (1989), Dynamics of meromorphic maps with polynomial Schwarzian derivative, Ann. Sci. Ecole Norm. Sup. (4) 22: 55-81. MR 90e:58071

[6] Douady, A. and Hubbard, J. (1985), On the dynamics of polynomial-like mappings, Ann. Sci. Ec. Norm. Sup. 18, 287-344. MR 87f:58083

[7] DuVal, P. (1973), Elliptic Functions and Elliptic Curves, Cambridge University Press. MR 52:417

[8] Eremenko, A. E., Lyubich, M. Y. (1992), Dynamical properties of some classes of entire functions, A. Inst. Fourier (Grenoble) 42: 989-1020. MR 93k:30034

[9] Hawkins, J., Koss, L. (2002), Ergodic properties and Julia sets of Weierstrass elliptic functions, Monatsh. Math. 137 no. 4: 273-300. MR 2003j:37066

[10] , (2003), Connectivity of Julia sets of elliptic functions, preprint.

[11] Jones, G., Singerman, D. (1997), Complex Functions: An algebraic and geometric viewpoint, Cambridge Univ. Press. MR 89b:30001

[12] Keen, L., Kotus, J. (1997), Dynamics of the family $\lambda \tan z$, Conform. Geom. Dyn. 1: 28-57. MR 98h:58159

[13] , (1999), Ergodicity of some classes of meromorphic functions, Ann. Acad. Sci. Fenn. Math. 24: 133-145. MR 2000a:30049

[14] Kotus, J., Urbanski, M. (2003), Hausdorff dimension and Hausdorff measures of Julia sets of elliptic functions, Bull. London Math. Soc. 35, 269-275. MR 2003j:37067

[15] Lyubich, M. (1987), The measurable dynamics of the exponential map, Siberian J. Math 28: 111-127. MR 89d:58071

[16] Mañé, R., Sad, P., Sullivan, D. (1983), On the dynamics of rational maps, Ann. Sci. Ecole Norm. Sup. $164^{e}$ série: 193-217. MR 85j:58089

[17] Mathematica, Wolfram Research, Inc. 1988-2002.

[18] McMullen, C. T. (1987), Area and Hausdorff dimension of Julia sets of entire functions. Trans. Amer. Math. Soc. 300: 329-334. MR 88a:30057 
[19] _ (2000), The Mandelbrot set is universal, The Mandelbrot Set, Theme and Variations, London Math Soc. Lecture Notes 274, Cambridge Univ. Press, 1-17. MR 2002f:37081

[20] McMullen, C. T., Sullivan, D. (1998), Quasiconformal homeomorphism and dynamics, III: The Teichmüller space of a holomorphic dynamical system. Adv. in Math. 135: 351-395. MR 99e:58145

[21] Milne-Thomson, L. (1950), Jacobian Elliptic Function Tables, Dover Publications, Inc.

Department of Mathematics, University of North Carolina at Chapel Hill, CB \#3250, Chapel Hill, North Carolina 27599-3250

E-mail address: jmh@math.unc.edu

Department of Mathematics and Computer Science, Dickinson College, P.O. Box 1773, Carlisle, Pennsylvania 17013

E-mail address: koss@dickinson.edu 